\title{
Review on X-ray Detectors Based on Scintillators and CMOS Technology
}

\author{
Jose G. Rocha ${ }^{1 *}$ and Senentxu Lanceros-Mendez ${ }^{2}$ \\ ${ }^{1}$ Algoritmi Research Center, University of Minho, Campus de Azurem, 4800-058 Guimaraes, Portugal. ${ }^{2}$ Center of \\ Physics, University of Minho, Campus de Gualtar, 4710-057 Braga, Portugal \\ Received: May 27, 2010; Accepted: June 29, 2010; Revised: September 3, 2010
}

\begin{abstract}
This article describes the theoretical basis, design and implementation of X-ray microdetectors based on scintillating materials and CMOS technology. The working principle of such microdetectors consists in the absorption of $\mathrm{X}$-rays by scintillators, which produce visible light. The visible light is then detected and converted into electric signals by means of photodetectors. In order to understand such detectors, several issues related to its implementation are presented in this article, namely:

Production of X-rays and interaction between them and matter - the first step necessary to the detection of X-rays is that they must be absorbed by some material, in this case by a scintillator;

Radiation detectors - there are several types of detectors, namely: pn junctions, photoconductors, based on thermal effects and scintillators;

Fabrication of scintillator arrays - after the X-ray radiation is absorbed by a scintillator, this material emits visible light whose intensity is proportional to the total energy of the absorbed X-rays;

Optical interfaces between scintillators and photodetectors - the visible light generated by scintillators must arrive to the photodetectors, so, it is necessary to have an interface between the scintillators and the photodetectors that ideally does not introduce losses;

Photodetectors and interface electronics - the visible light is absorbed by the photodetectors and converted into electrical signals, which are finally converted into digital images by means of interface electronics. The article presents some promising patents on X-ray detectors based on scintillators and CMOS technology.
\end{abstract}

Keywords: X-rays, scintillators, digital radiography, CMOS imaging.

\section{INTRODUCTION}

In the years that preceded the discovery of X-rays, some physicists observed high-voltage electric discharges in vacuum tubes. In 1895, the German physicist Wilhelm Konrad Röentgen studied the same phenomenon in a Crookes tube [1] operating at high voltage, in a darkened room. Suddenly, he observed fluorescence (brightness) in a barium platinocyanide screen placed a few meters from the tube. He quickly concluded that the fluorescence was caused by an invisible, unknown radiation, which could completely pass through solid materials such as paper and wood, once they did not prevent the fluorescence when placed between the tube and the screen. He also verified that the radiation could be stopped by denser materials, such as lead.

Röentgen discovered the X-rays and at the same time, he discovered the first radiographic image detector. Barium platinocyanide is not used anymore, but the principles of fluorescence or scintillation are applied in about $95 \%$ of modern X-ray detectors. In some detectors, like traditional

*Address correspondence to these authors at the Algoritmi Research Center, University of Minho, Campus de Azurem, 4800-058 Guimaraes, Portugal; Tel: +351 253 510190; Fax: +351 253 510189;

E-mail: gerardo@dei.uminho.pt; Center of Physics, University of Minho, Campus de Gualtar, 4710-057 Braga, Portugal; Tel: +351 253 604073;

Fax: +351 253 678467; E-mail: lanceros@ fisica.uminho.pt radiographic films, scintillators are not essential, but they are generally used. In some imaging systems, such as computerized tomography and nuclear medicine imaging, an essential characteristic of the scintillators is used to advantage: they produce a flash of light proportional to the energy of each X-ray photon that interacts with them. A system for providing multiple images in an imaging unit is disclosed by Erwen et al. in 2010 [2].

\subsection{X-Ray Nature}

An X-ray beam belongs to a group of radiations of the same nature as infrared, visible light, ultraviolet, radio waves and other types of radiation energy [3]. All these energy forms are classified as electromagnetic radiations, that is, fluctuations of electric and magnetic fields. The electric and magnetic fields change perpendicularly to the propagation direction, as well as between themselves. Its speed is constant and equal to $299792458 \mathrm{~ms}^{-1}$. This is known as speed of the light in vacuum.

All types of electromagnetic radiation differ only in the frequency of oscillation, $v$, and/or in the wavelength, $\lambda$. These two quantities are related by the constant speed of the light, $c$ :

$v=c / \lambda$. 
The electromagnetic radiation can also be understood as a set of particles that travel in space at the speed of light, called quanta or photons, each one having a fixed amount of energy given by:

$E_{\gamma}=h v$,

where $h=6.63 \times 10^{-34}$ Js is the Planck's constant. They also have momentum given by:

$p=E_{\gamma} / c$.

An electromagnetic radiation is fully characterized by its wavelength, its frequency or its energy, once these quantities are related. The X-rays are usually characterized by their energy, measured in electron-Volt $(\mathrm{eV})$, where $1 \mathrm{eV}$ is the energy that an electron acquires when it is speed up by a potential difference of 1 Volt and is approximately $1.6 \times 10^{-19} \mathrm{~J}$. The X-rays used in medical imaging usually are in the range from $15 \mathrm{keV}$ to $120 \mathrm{keV}$.

\subsection{Production of $X$-Rays}

There are some devices used for the production of X-ray radiation, namely the $\mathrm{X}$-ray tube, the synchrotron, and the free electron laser. In medical imaging, the X-ray tube is the most widely used source, whereas in radiotherapy, the synchrotron is also used. The working principle of the X-ray tube is described in the following sections. Tutomu et al. described the function of X-ray tube in patents no. US7773726 (2010)[4].

\subsubsection{X-Ray Tube}

When electrons are speed up to more than $5 \mathrm{keV}$ and strike directly in the surface of a target, X-rays are emitted. The X-rays are mainly originated in the fast deceleration of the electrons, when they interact directly with the nuclei of the atoms of the target. This principle of production of Xrays is called bremsstrahlung, a German word which means braking radiation. Fig. (1) shows a schematic diagram of an X-ray tube.

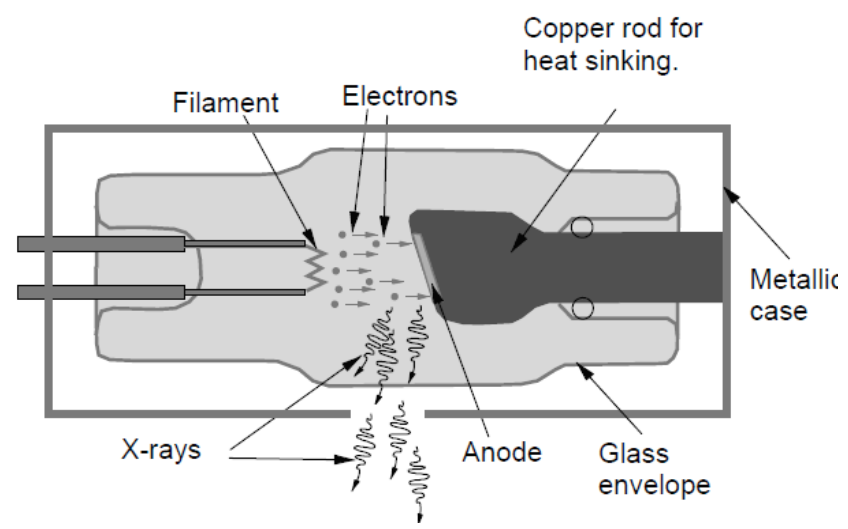

Fig. (1). Schematic diagram of an X-ray tube.

Basically, an X-ray tube is composed by:

A filament that is heated by a low-voltage electric current. This filament delivers electrons by thermionic emission.
- A glass cover to keep the device in vacuum conditions.

- An anode that converts the energy of the electrons into $\mathrm{X}$-rays.

- A case with a window transparent to X-rays, for the outgoing of the X-rays.

When an electrical current flows through the filament, electrons are released by thermionic emission. They are then sped up by a potential difference, of the order of tens or hundreds of kilovolts, which is applied between the filament and the anode. The high-sped electrons, when striking in the anode, lose their energy in the form of heat and electromagnetic radiation. A small part of this radiation is in the spectral range of the X-rays, as will be shown in the following paragraphs.

\subsubsection{X-Ray Spectrum}

If electrons are speed up by a potential of $35 \mathrm{kV}$, when they strike in a molybdenum anode, the spectrum of Fig. (2) is produced. This spectrum is composed by two parts: the continuous spectrum of the braking radiation and the characteristic radiation spectrum of the molybdenum anode.

\subsubsection{Continuous Spectrum}

When a high-speed electron collides with the anode, several processes can occur: the most probable one is that the electron suffers a small elastic scattering, which corresponds to transference of energy to the anode, which normally appears under the form of heat. In the energy range used in medical imaging, about $99 \%$ of the electron energy is converted into heat. Its dissipation is one of the biggest technical problems of X-ray tubes.

Occasionally, the electron passes very close to an atomic nucleus, where it suffers a deflection, mainly due to the nuclear charge. The interaction of the electron with the atomic nucleus results in a change of its kinetic energy, which results in the emission of a photon in the spectral range of the X-rays. The energy lost by the electron can have a wide range of values, which justifies the continuous spectrum. The emission of X-rays can also occur after some elastic scattering interactions, which means that the X-rays are not always emitted from the surface of the anode. This factor, besides justifying the continuous spectrum of the emission of X-rays, also justifies its continuous spatial distribution.

\subsubsection{Characteristic Spectrum}

Overlapped to the continuous spectrum, characteristic peaks normally appear which are the result of the interaction of high-speed electrons with the ones of the atoms of the target. If a high-speed electron has enough energy, it can ionize an atom, removing one atomic electron from its orbital. In this case, the orbital in question will have a lack of an electron, i.e., a hole. Usually, this happens in the layers close to the nucleus. The hole will then be filled by another electron of a higher shell, which jumps to it. In this jump, the electron loses energy by releasing a photon. If the phenomenon occurs in the layers close to the nucleus, the released photon will be in the spectral range of the X-rays. 
As an example, a high-speed electron removes an electron of the $K$ shell of an atom. An electron from the $L$ or $M$ shells will fill the existing hole in $K$ shell. As the energy difference between electrons of $L$ or $M$ shells and $K$ shell is constant for each chemical element, all photons released in this way have the same energy. This explains the spectral peaks, where each one of them corresponds to a well-defined jump. The peaks shown in the spectrum of Fig. (2) correspond to jumps between $L_{I}, L_{I I}$ and $K$ shells in molybdenum.

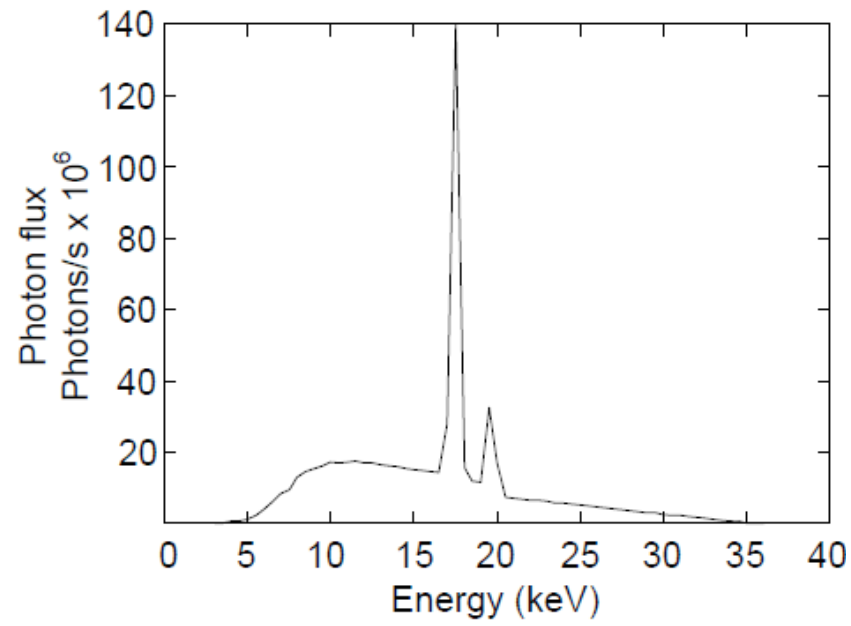

Fig. (2). X-ray spectrum produced by a molybdenum anode and a potential difference of $35 \mathrm{kV}$.

\subsection{Interaction Between $X$-Rays and Matter}

The operation of any X-ray detector depends basically on the way the radiation interacts with the material that constitutes it. Notwithstanding several interaction mechanisms of $\mathrm{X}$-rays and matter are known, only three of them are important in their measurement: photoelectric absorption, Compton scattering and production of electron-positron pairs. These processes are based on the partial or total transfer of the X-ray photon energy to an atom. As a result, the trajectory and the energy of the photon are drastically modified.

When a beam of X-rays passes through a body, along $x$ direction, after the distance $d x$ in its interior, the X-rays intensity decreases. The decrease in intensity is given by:

$\frac{d I}{d x}=-\mu I$,

where $I$ is the intensity of the beam and $\mu$ is the coefficient of linear absorption of the material. Integrating along the thickness of the body, gives:

$I=I_{O} e^{-\mu x}$,

where $I_{o}$ is the initial intensity of the beam. Usually, instead of $\mu$, the mass absorption coefficient $\mu / \rho$ is used (coefficient of linear absorption per unit of density) and equation (5) becomes:

$I=I_{O} e^{(-\mu / \rho) \rho x}$.
The mass absorption coefficient $\mu / \rho$ is related to the cross-sections of the interaction processes of the X-rays with matter, according to:

$\mu / \rho=\frac{N_{A V}}{A} \sum_{i} \sigma_{i}$,

where $\sigma_{i}$ is the atomic cross-section of the interaction process $i, A$ is the relative atomic mass of the atom with which the interaction occurs and $N_{A V}$ is Avogadro's number $\left(6.022 \times 10^{23}\right)$. For energies below $100 \mathrm{keV}$, the dominant interaction is the photoelectric absorption [5], which can be represented by:

$\gamma+$ atom $\rightarrow$ atom $^{+}+e^{-}$

where $\gamma$ represents a photon and $e^{-}$an electron. In the medium range of energies (close to $1 \mathrm{MeV}$ ), the Compton effect is dominant. The Compton effect is basically the scattering of a photon when interacting with an electron [5]:

$\gamma+e^{-} \rightarrow \gamma+e^{-}$.

At very high energies (far above $1 \mathrm{MeV}$ ), the cross-section for the production of electron-positron pairs is the most significant [5],

$\gamma+$ nucleus $\rightarrow e^{+}+e^{-}+$nucleus.

In this case, $e^{+}$represents a positron.

\subsubsection{Photoelectric Effect}

Atomic electrons can absorb the total energy of a photon, whereas for free electrons, due to momentum conservation, such is not possible. The absorption of a photon by an atomic electron requires a third collision entity, which in this in case is the nucleus of the atom. The cross-section for the absorption of a photon of energy $E$ in the $K$ shell of an atom is particularly high $(\approx 80 \%$ of the total cross-section), due to the proximity of the third collision entity, the atomic nucleus, which absorbs the recoil momentum. The total cross-section for the photoelectric effect in the non-relativistic range far from absorption edges is given by the Born approach [6]:

$\sigma_{\text {photo }}^{K}=\left(\frac{32}{\varepsilon^{7}}\right) \alpha^{4} Z^{5} \sigma_{T h}^{e}$,

where:

$\varepsilon=E_{\gamma} / m_{e} c^{2}$,

is the reduced energy of the photon, $E_{\gamma}$ is the photon energy, $m_{e}$ is the electron mass, $Z$ is the atomic number and $c$ is the speed of light. $\sigma_{T h}^{e}$ is the Thomson cross-section for elastic collisions between photons and electrons, given by:

$\sigma_{T h}^{e}=\frac{8}{3} \pi r_{e}^{2}=6.65 \times 10^{-29} \mathrm{~m}^{2}$,

From classical theory, the electron radius is given by:

$r_{e}=\frac{1}{4 \pi \varepsilon_{O}} \frac{e^{2}}{m_{e} c^{2}}=2.81794 \times 10^{-15} \mathrm{~m}$, 
where $e$ is the electron charge and $\varepsilon_{o}$ is the vacuum permittivity $\left(8.85 \times 10^{-12} \mathrm{~F} / \mathrm{m}\right)$. The fine-structure constant is given by:

$\alpha=\frac{e^{2}}{4 \pi \varepsilon_{O} \hbar c}=7.29735 \times 10^{-3}$,

where $\hbar=h / 2 \pi$ is the reduced Planck constant.

Close to the absorption edges, the dependence between the cross-section and the photon energy is modified by a function $f\left(E_{\gamma}, E_{\lambda}^{e d g e}\right)[6]$. For high energies the dependence between cross-section and energy for the photoelectric effect is:

$\sigma_{\text {photo }}^{K}=4 \pi r_{e}^{2} Z^{5} \alpha^{4} \frac{1}{\varepsilon}$.

In equations (11) and (16), the dependence between the cross section and the atomic number is $Z^{5}$. This is an indication that interactions between photons and isolated atomic electrons do not occur. Actually, the dependence between the cross-section and the atomic number shows that $\sigma_{\text {photo }}$ is a more complex function of $Z$. In the energy range between $100 \mathrm{keV}$ and $5 \mathrm{MeV}$, the exponent of $Z$ ranges between 4 and 5 .

As a consequence of the photoelectric effect in an inner shell of an atom ( $K$ shell, for example), some secondary effects can occur. If the hole left after the interaction between the photon and the electron is filled by another electron of a higher shell, the energy difference can be released under the form of a characteristic X-ray photon, which in turn can be absorbed by another electron of the same or neighboring atoms. If the energy is higher than the binding energy of the electronic shell of the atom in question, another electron can leave the atom (Auger effect) [7]. The energy of these Auger electrons is necessarily smaller than the energy of the primary electron. For example, if the photoionization occurs in $K$ shell with binding energy $E_{K}$, and the hole is filled by an electron of $L$ shell, whose energy is $E_{L}$, the amount of energy $E_{K}-E_{L}$ will be released. This energy, in turn, can be transferred to another electron of $L$ shell. If $E_{K}-E_{L}>E_{L}$, this electron can leave the $L$ shell as an Auger electron, with kinetic energy of $E_{K}-2 E_{L}$.

\subsubsection{Compton Effect}

The Compton effect describes the scattering of photons when interacting with free electrons. In practice, one can use the relations established here for atomic electrons although the theory is developed for free electrons. In the mathematical model of this interaction process, the binding energy of electrons to atoms is normally disregarded. The total cross-section, for each electron, for the Compton scattering is given by the Klein-Nishina formula [8]:

$\sigma_{C}^{e}=2 \pi r_{e}^{2}\left\{\left(\frac{1+\varepsilon}{\varepsilon^{2}}\right)\left[\frac{2(1+\varepsilon)}{1+2 \varepsilon}-\frac{1}{\varepsilon} \ln (1+2 \varepsilon)\right]+\frac{1}{2 \varepsilon} \ln (1+2 \varepsilon)-\frac{1+3 \varepsilon}{(1+2 \varepsilon)^{2}}\right\}$,

where $\varepsilon$ is given by equation (12) and $r_{e}$ by equation (14).
The Compton scattering for an atom as a whole is proportional to the atomic number:

$\sigma_{c}^{a t o m}=Z \sigma_{c}^{e}$

The relationship between the energies of scattered and incident photons is given by:

$\frac{E_{\gamma}^{\prime}}{E_{\gamma}}=\frac{1}{1+\varepsilon\left(1-\cos \theta_{\gamma}\right)}$,

where $\theta_{\gamma}$ is the scattering angle of the photon in the referential of the laboratory Fig. (3).

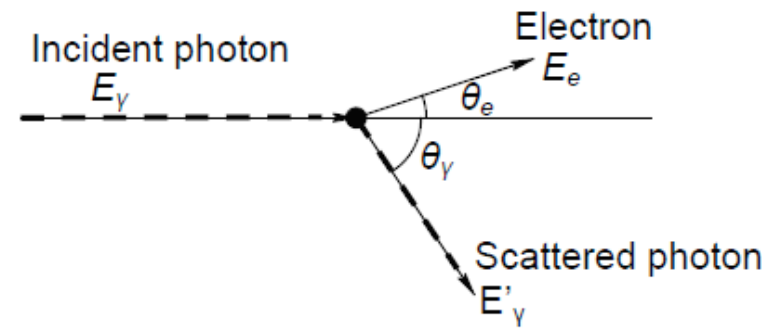

Fig. (3). Kinematic variables of the Compton scattering process.

When $\theta_{\gamma}=180^{\circ}$ (backscattering) the energy transferred to the electron reaches its maximum value. The relationship between the energies of scattered and incident photons is then given by:

$\frac{E_{\gamma}^{\prime}}{E_{\gamma}}=\frac{1}{1+2 \varepsilon}$.

The scattering angle of the electron, relatively to the direction of the incident photon is given by:

$\cot \theta_{e}=(1+\varepsilon) \tan \frac{\theta_{\gamma}}{2}$.

Due to momentum conservation, this angle cannot be larger than $\pi / 2$. In Compton scattering only a portion of the photon energy is transferred to the electron. The crosssection of energy scattering can then be defined as:

$\sigma_{c s}=\frac{E_{\gamma}^{\prime}}{E_{\gamma}} \sigma_{c}^{e}$

and the absorption cross-section is defined as:

$\sigma_{c a}=\sigma_{c}^{e}-\sigma_{c s}$.

Equation (23) is important in absorption processes and is related to the probability that the kinetic energy $E_{c i n}=E_{\gamma}-E_{\gamma}^{\prime}$ be transferred to an electron.

Compton scattering occurs not only with electrons, but also with other charged particles. However, the interaction with atomic electrons is the most important for the operation of radiation detectors.

\subsubsection{Production of Electron-Positron Pairs}

The production of an electron-positron pair in the electric field of the nucleus of an atom is only possible if the energy 
of the photon exceeds a certain threshold, given by the rest masses of the electron plus the positron, multiplied by $c^{2}$, added to the recoil energy that is transferred to the nucleus of the atom. From the conservation of energy and momentum, the energy threshold for the production of an electronpositron pair is [8]:

$E_{\gamma} \geq 2 m_{e} c^{2}+2 \frac{m_{e}^{2}}{m_{\text {nucleus }}} c^{2}$.

As the electron mass $\left(m_{e}\right)$ is far smaller than the mass of the nucleus $\left(m_{\text {nucleus }}\right)$, the expression of the energy threshold can be approximated by:

$E_{\gamma} \geq 2 m_{e} c^{2}$.

On the other hand, if the interaction takes place in the electric field of an electron, the expression for the energy threshold becomes:

$E_{\gamma} \geq 4 m_{e} c^{2}$

The probability of the production of an electron-positron pair occurring in the field of the electron is, however, far smaller than the probability of occurring in the field of the nucleus [8].

In the case where the nuclear charge is not shielded by atomic electrons (the photon passes close to it) and

$\varepsilon<\frac{1}{\alpha Z^{1 / 3}}$

the cross-section for electron-positron pair production is given by [9]:

$\sigma_{\text {pair }}=4 \alpha r_{e}^{2} Z^{2}\left(\frac{7}{9} \ln 2 \varepsilon-\frac{109}{54}\right)$.

At low energies, the photon must pass relatively close to the nucleus so that a pair production can take place, which means that the photon will see the nucleus uncovered by electrons.

In the case of high photon energy, that is,

$\varepsilon>>\frac{1}{\alpha Z^{1 / 3}}$,

and the nucleus is shielded by electrons, the cross-section for the production of electron-positron pairs is given by:

$\sigma_{\text {pair }}=4 \alpha r_{e}^{2} Z^{2}\left(\frac{7}{9} \ln \frac{183}{Z^{1 / 3}}-\frac{1}{54}\right)$.

In this case, the fact that the nuclear charge is covered by electrons must be taken into account. For high energy photons, the cross-section for the production of electronpositron pairs tends to a value that is independent of the energy of the incident photon, as it can be seen in equation (28). As the term 1/54 in equation (28) is far smaller than the other, it can be disregarded, giving the following approximated equation:

$\sigma_{\text {pair }} \approx \frac{7}{9} 4 \alpha r_{e}^{2} Z^{2} \ln \frac{183}{Z^{1 / 3}}$.
Notice that the production of an electron-positron pair cannot happen in vacuum, due to momentum conservation. The photon has a momentum $p=E_{\gamma} / c$, and as $E_{\gamma} \geq 2 m_{e} c^{2}$ (equation (25)), $\quad p \geq 2 m_{e} c$, that is, the electron and the positron would move at speeds superior to the one of the light, which is impossible. Therefore it is necessary that another particle, in whose field the formation of pairs occurs, to receive the difference of momentum.

\subsubsection{Other Processes of Interaction Between X-Rays and Matter}

Beyond the three previously described processes, there are others whose interest for the implementation of detectors is smaller, namely:

\section{Coherent or Rayleigh Scattering}

In opposite to the Compton scattering of photons in individual electrons, the Rayleigh scattering occurs only in electrons that are part of an atom. Once the recoil momentum in Rayleigh interaction is absorbed by the atom as a whole, the loss of energy of the photon is insignificant and the scattering angle is small. Due to the fact that the effect in the energy or direction of the photon is minimal, usually it is a common procedure to ignore Rayleigh scattering in the calculations of interactions between X-rays and matter [10].

\section{Photonuclear Absorption}

The absorption of a photon by an atomic nucleus normally results in the emission of one or more neutrons and/or protons. This interaction can contribute $5 \%$ to $10 \%$ to the total cross-section of interaction with photons in a relatively narrow range of energies, normally between 5 $\mathrm{MeV}$ and $40 \mathrm{MeV}$, depending on the nucleus in question [11 - 15]. The effects of this interaction can be observed in measurements of the total attenuation coefficient [16]. However, there is an irregular dependence between this cross-section and the atomic number or the atomic mass, and there are not theoretical models comparable to the ones of the other cross-sections.

\section{Elastic Nuclear Scattering}

This is an effect analogous to Compton scattering, but produced by the nucleus. In this process, a photon interacts with the nucleus in such a way that it is absorbed and reemitted with the same energy [12].

\section{Inelastic Nuclear Scattering}

The nucleus of an atom is excited to a higher energy level by the absorption of a photon. The excited nucleus then releases another photon with equal or smaller energy than the first one [12].

\section{Delbrück Scattering}

The phenomenon of scattering of a photon by the electric field of the nucleus is called Delbrück scattering. This phenomenon can be understood as the formation of a virtual electron-positron pair in the field of the nucleus followed by its annihilation $[17,18]$. 


\subsubsection{Total Cross Section of Absorption of Photons}

The mass absorption coefficient, which is related with the cross-sections for the interaction processes, according to equation (7), is shown in Fig. (4) for cesium iodide. In this figure, it can be verified that in the energy range of interest for X-ray detectors (represented in a gray background), the most important processes of interaction are the photoelectric effect and Compton scattering.

Since Compton scattering has a special importance in the interactions between photons and electrons, due to the fact that only a portion of the energy is transferred between them, the mass attenuation coefficient and the mass absorption coefficient must be distinguished [8]. The mass attenuation coefficient $\left(\mu_{c s}\right)$ is related to the cross section of the energy scattering of Compton $\left(\sigma_{c s}\right)$ (equations (22) and (7)). In an analogous way, the mass absorption coefficient $\left(\mu_{c a}\right)$ is calculated from the cross-section of energy absorption of Compton $\left(\sigma_{c a}\right)$ (equations (23) and (7)).

The mass absorption coefficient of a composite or mixture of elements can be calculated from the individual coefficients of the elements from:

$$
(\mu / \rho)_{c}=\sum_{i} w_{i}(\mu / \rho)_{i}
$$

where $w_{i}$ represents the ratio of mass of element $i$ in the composite or the mixture. In the example of Fig. (4), iodine with atomic number 53 and relative atomic mass of 126.9 contributes with a ratio of mass of $48.8 \%$, whereas cesium, with atomic number 55 and relative atomic mass of 132.9, contributes with a ratio of mass of $51.2 \%$.

\section{RADIATION DETECTORS}

The output signal of a radiation detector results from the ionization and/or excitation of atoms produced by the previously described interactions, which occur in the detection medium. The output signal of the detector can be produced by primary ionizations, thermal changes or scintillations that afterward are converted into electric signals. Usually, the output electric signal must be close to a value proportional to the energy that falls upon the detection medium. There are several types of detectors, namely: pn junctions, photoconductors, based on thermal effects and scintillators. Each method has its own advantages and disadvantages.

\subsection{Detectors Based in pn Junctions}

The simplest model of radiation detector is based on a pn junction, usually implemented in amorphous silicon substrates. X-ray photons that are absorbed in the depletion region of the junction create electron-hole pairs, which are separated by the electric field that exists in this region. Amorphous silicon is often preferred instead of crystalline silicon in high-area detectors (up to $50 \mathrm{~cm} \times 50 \mathrm{~cm}$ ) for digital radiography. This is mainly due to the enormous difficulty in manufacturing devices of those dimensions in crystalline silicon. The main advantage of this method is that a little more than $1 \mathrm{eV}$ of energy is enough to produce an electron-hole pair. Unfortunately, the X-ray absorption power of silicon is much reduced. For example, a silicon detector with thickness of $525 \mu \mathrm{m}$ would only absorb approximately $2.2 \%$ of $100 \mathrm{keV}$ X-rays. In order to increase the absorption to near $50 \%$, the same detector must have 16 $\mathrm{mm}$ of thickness, which is not practical.

\subsection{Detectors Based in Photoconductors}

The photoconductor method uses materials with higher absorption power than silicon and whose conductivity changes with the amount of absorbed radiation.

The best known photoconductor is probably amorphous selenium. When compared with silicon, its X-ray absorption is a little better (the thickness of $525 \mu \mathrm{m}$ would absorb approximately $12.7 \%$ of $100 \mathrm{keV}$ X-rays), but it needs about $50 \mathrm{eV}$ for producing an electron-hole pair. Another disadvantage is that it needs a relatively high bias voltage (about $10 \mathrm{~V} / \mu \mathrm{m}$ ) to work properly [19].

Other promising photoconductors are based on CdTe, $\mathrm{CdZnTe}, \mathrm{HgI}_{2}$ and $\mathrm{PbI}_{2}[20-22]$. Due to the fact that they are constituted of heavy elements, they have high X-ray absorption power. However, they show almost the same disadvantages as amorphous selenium.

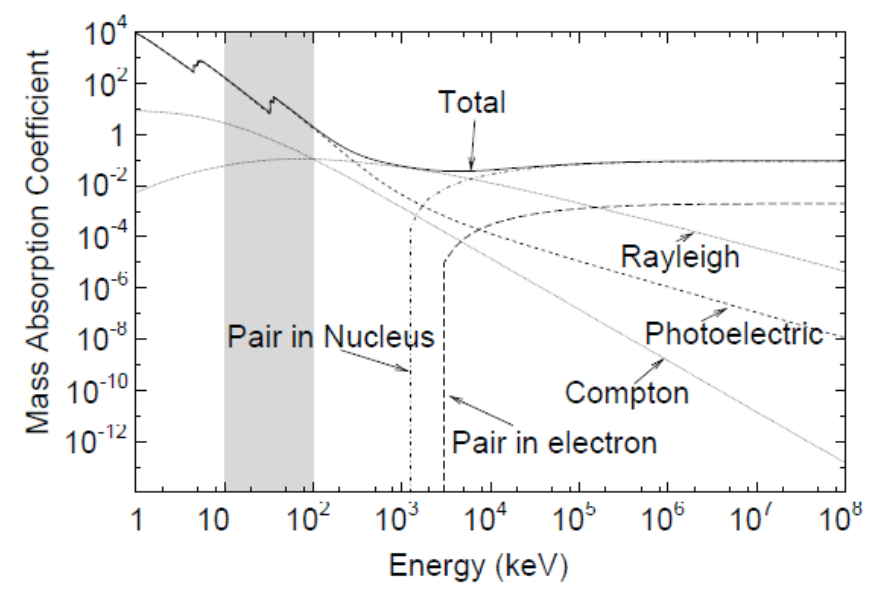

Fig. (4). Mass absorption coefficients of cesium iodide. The energy range of interest for X-ray detectors based in scintillators is represented in a gray background. 


\subsection{Detectors Based in Thermal Effects}

The working principle of this kind of detector is based on the Seebeck effect. It consists of the detection of the temperature increase caused by the absorption of X-rays by a material of high atomic number and high density. The junction of two thermoelectric materials, with different Seebeck coefficients, is used to detect the temperature increase. Fig. (5) shows the basic structure of such detector. As target, copper can be used and as thermoelectric materials, $\mathrm{Sb}_{2} \mathrm{Te}_{3}$ of $p$ type and the $\mathrm{Bi}_{2} \mathrm{Te}_{3}$ of $\mathrm{n}$ type materials can be used [23]

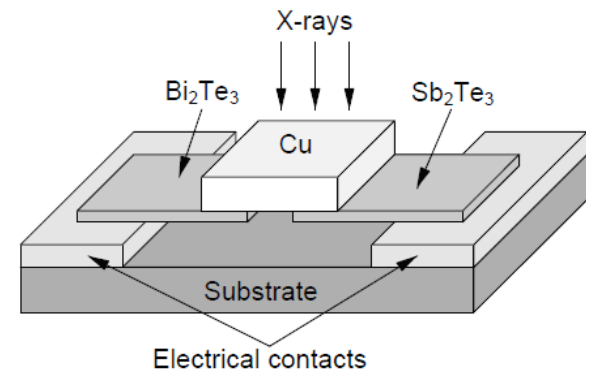

Fig. (5). Structure of an X-ray detector based in thermal effects.

This method does not need bias voltages, as the previous ones, since the output signal appears as an electric voltage. As main disadvantage, it can be pointed out the frequency response, which is very reduced, of the order of tenths of $\mathrm{Hz}$, or at most of some $\mathrm{Hz}$.

\subsection{Detectors Based in Scintillators}

This method uses a material that absorbs the radiation and converts its energy into visible light, which is then detected by a conventional photodetector. A good scintillator must be constituted by chemical elements of high atomic number and have high density, in order to absorb the X-rays. It must further produce a high number of visible light photons for each absorbed X-ray photon.

This approach seems to be the best, since scintillators have high X-ray absorption power, they do not need bias voltages and they have very high frequency responses (usually in the $\mathrm{GHz}$ range).

Fig. (6) shows the structure of an X-ray image detector based on scintillators. It consists basically of a scintillating layer placed on top of a matrix of photodetectors.

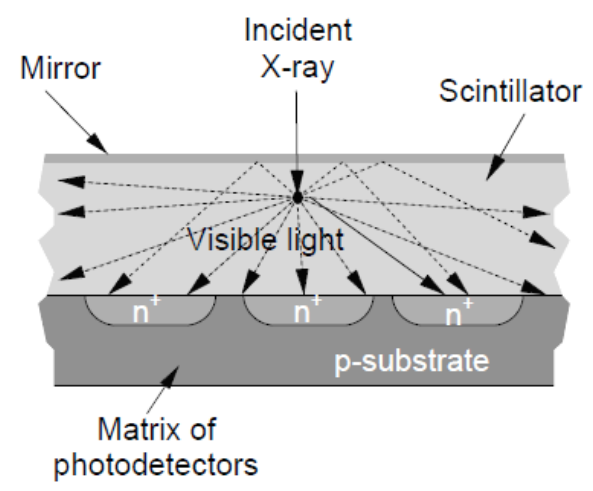

Fig. (6). Basic structure of a X-ray detector based on scintillators.
This method, being relatively simple, has some limitations in spatial resolution, due to the restrictions imposed by the height of the scintillator. On one hand, it is desirable to have a a large height of the scintillator layer, so that more $\mathrm{X}$ ray photons are absorbed. On the other hand, when increasing the height of the scintillator, the amount of light produced in one pixel that reaches its neighbors is higher, that is, the spatial resolution decreases [24].

A method to increase the spatial resolution, without decreasing the height of the scintillator, consists of using an individual scintillator for each pixel, separated by layers of a reflector material, as is shown in Fig. (7).

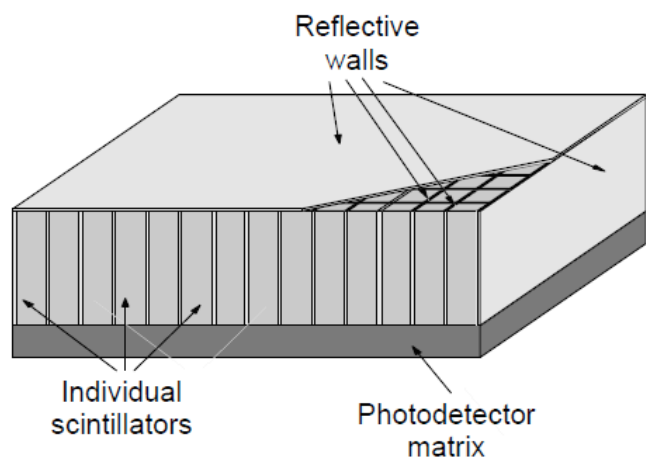

Fig. (7). Schematic representation of a structure based on light guides that are used to increase the spatial resolution of the detectors of radiation based on scintillators.

This method will be detailed in the following sections.

\section{X-RAY DETECTORS BASED ON SCINTILLATORS}

X-ray detectors based on scintillators, firstly convert Xray energy into visible light, which is then converted into electrical signals by means of photodetectors. Fig. (8) shows a schematic diagram of a detector of this kind, where the scintillator is the thallium doped cesium iodide (CsI:Tl), which is embedded inside reflective layers of aluminum. Aluminum is a material of low atomic number and low density, allowing the penetration of X-rays. On the other hand, it shows high reflectivity for visible light, guiding it to the corresponding photodetector.

The most critical steps of this process that can influence the efficiency and the signal to noise ratio of the detector are:

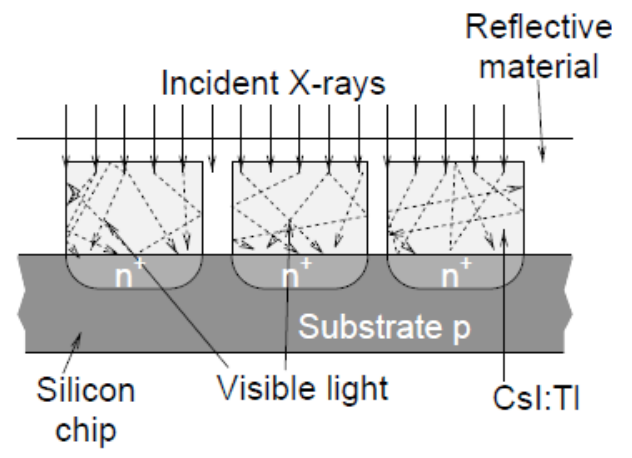

Fig. (8). Schematic diagram of an X-ray imaging detector based in scintillators. 
- Transmission of the X-rays through the aluminum reflective layer.

- Absorption of X-rays by the scintillator and their conversion into visible light.

- Reflection of visible light by the reflective layers.

- Transmission of visible light to the photodetector.

- Detection of visible light and its conversion into electrical signals.

Some considerations must be taken into account before the explanation of each step:

1. The most common noise sources in X-ray imaging detectors are the photon noise, the fixed pattern noise and the noise of the readout electronics [25]. Fixed pattern noise is present in all detector matrices and can be cancelled by using gain maps [26]. The readout electronic noise depends mainly on the electronic configuration and usually is less important than the photon noise.

2. The photon noise, caused by the quantized nature of Xrays, is the fundamental limitation of the signal to noise ratio of the detector. Several theoretical and experimental analyses demonstrate that the intrinsic photon noise of counting the events from an X-ray beam is random and follows a Poisson distribution, that is, the standard deviation, $\sigma_{p r x}$, is equal to the square root of the mean number of detected photons, $m_{p r x}[27,28]$, i. e.:

$\sigma_{\text {prx }}=\sqrt{m_{\text {prx }}}$

Fig. (9) shows the spectral distribution of the photons produced by an X-ray tube with molybdenum anode, when it is powered by a $35 \mathrm{kV}, 1 \mathrm{~mA}$ source, measured by a detector of $1 \mathrm{~mm}^{2}$ of area, placed at $0.5 \mathrm{~m}$ from the anode [29].

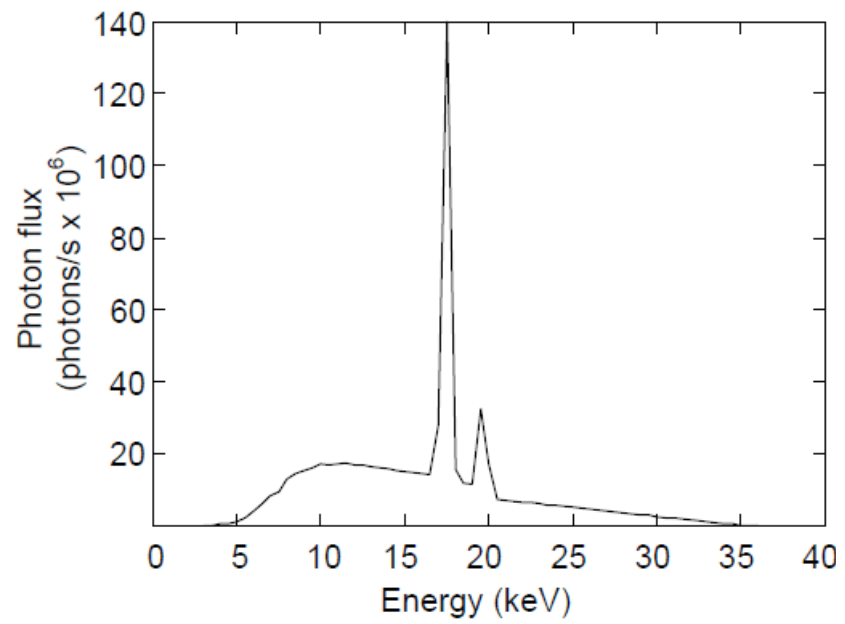

Fig. (9). Spectrum of the radiation produced by an X-ray tube with molybdenum anode, powered at $35 \mathrm{kV}, 1 \mathrm{~mA}$. The signal is measured by a detector of $1 \mathrm{~mm}^{2}$ area, placed at $0.5 \mathrm{~m}$ from the anode.

Fig. (10) shows the corresponding signal to noise ratio, which is calculated, for each energy, as the ratio between the mean number of photons and its standard deviation, for a unit exposure time.

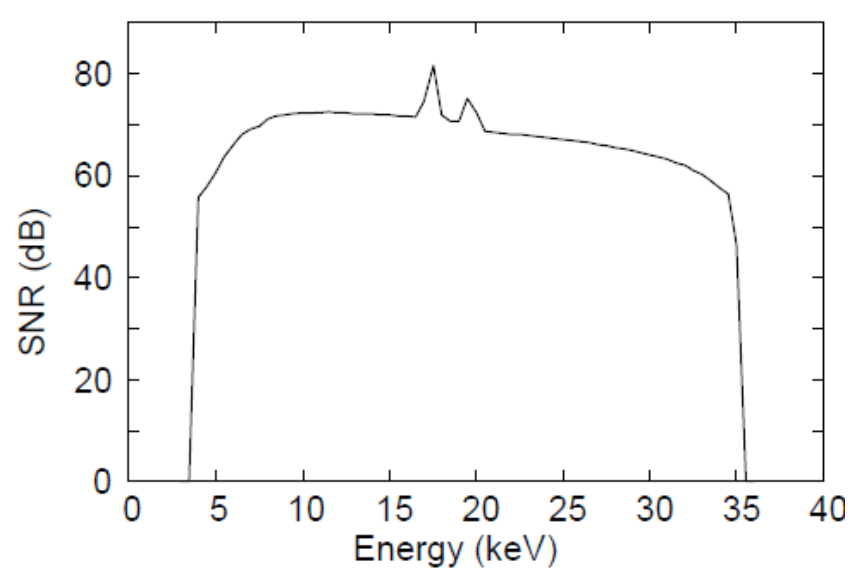

Fig. (10). Signal to noise ratio of the radiation produced by an Xray tube with a molybdenum anode, when powered by $35 \mathrm{kV}, 1$ $\mathrm{mA}$. The signal is measured by a detector of $1 \mathrm{~mm}^{2}$ area, placed at $0.5 \mathrm{~m}$ from the anode.

As mentioned before, the photons coming from the X-ray tube must follow several steps before being detected. These steps will be described in the following sections.

\subsection{Transmission of the $X$-Rays Through the Aluminum Reflective Layer}

When an X-ray beam penetrates some material, it is absorbed according to equation (5). Fig. (11) shows the Xrays percentage transmitted by aluminum layers of different thicknesses, between $10 \mu \mathrm{m}$ and $500 \mu \mathrm{m}$. As it can be observed, there is a reduction of the transmissivity with increasing the thickness of the layer.

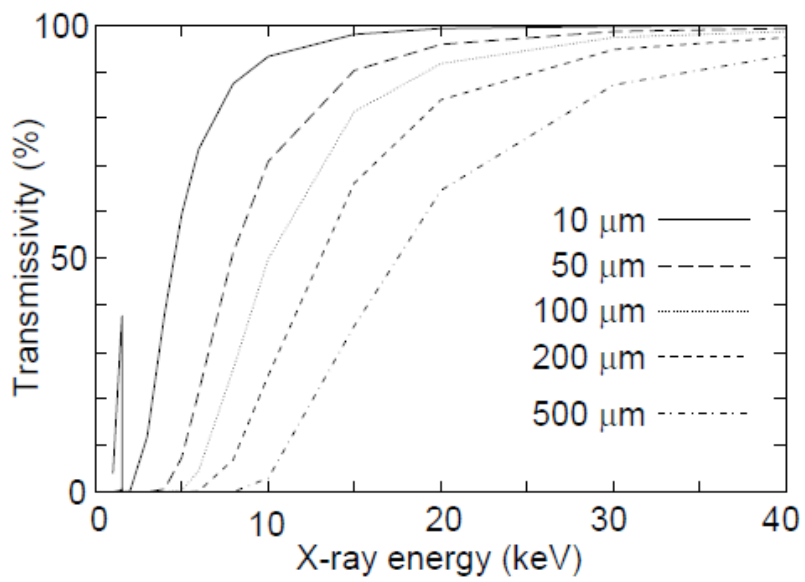

Fig. (11). Transmissivity to X-rays of different aluminum thicknesses.

\subsection{Absorption of $X$-Rays by the Scintillator and Their Conversion Into Visible Light}

The absorption of X-rays by the scintillator depends on the laws described in previous sections. However, scintillators are formed by composites or mixtures of some elements, therefore, the mass absorption coefficient must be calculated from the individual elements that form the composite, using equation (29). As was seen for CsI:Tl, 
iodine with atomic number 53 and relative atomic mass of 126.9 contributes with a fraction of mass of $48.8 \%$, whereas cesium, with atomic number 55 and relative atomic mass of 132.9, contributes with a fraction of mass of $51.2 \%$. Thallium concentration in the CsI:Tl is of about $0.02 \%$ to $0.03 \%$ [30], and its contribution for the absorption of rays- $\mathrm{x}$ is negligible.

Fig. (12) shows the percentage of X-rays that is absorbed by CsI:Tl scintillators with thicknesses between $100 \mu \mathrm{m}$ and $900 \mu \mathrm{m}$. As expected, the absorption increases with thickness.

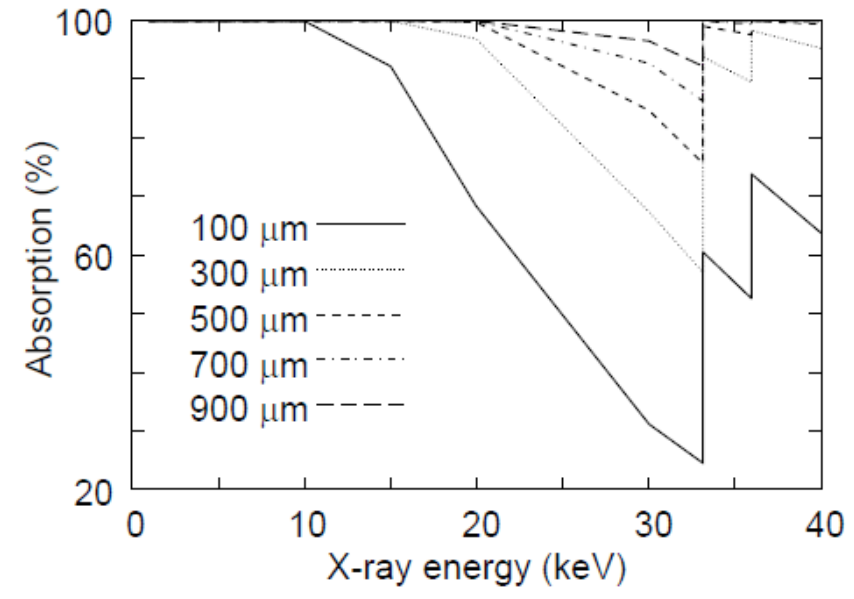

Fig. (12). Absorption of X-rays by CsI:T1 scintillators of different thicknesses.

The scintillator will convert X-rays into visible light. In the case of CsI:Tl, about 65900 visible photons (wavelength close to $560 \mathrm{~nm}$ ) are produced for each $1 \mathrm{MeV}$ of absorbed energy, at room temperature [31]. This means that for each photon of $1 \mathrm{MeV}$, a random number of visible photons is produced, whose mean is 65900 .

Therefore, the average amount of visible light produced, $L_{R}(E)$, at a given X-ray energy, for a unit time, is obtained from the product of five factors:

1. Number of X-ray photons that fall upon the detector, $N$, which is random,

2. Transmissivity of aluminum, $\operatorname{Tr}_{a l}$ Fig. (11),

3. Absorption of the scintillator, $A b_{s c}$ Fig. (12),

4. 65900 photons $/ \mathrm{MeV}$, which is the mean of a random process,

5. Energy of the incident X-ray photon, $E$, in $\mathrm{MeV}$.

That is:

$L_{R}(E)=N \times T r_{a l} \times A b_{s c} \times 65900 \times E$.

In this case, the variance of the produced visible photon distribution is given by the product of the average value of $\mathrm{X}$-ray photons absorbed by the scintillator by the square of the number of visible photons produced by each X-ray::

$\sigma_{L R}^{2}(E)=N \times T r_{a l} \times A b_{s c}(65900 E)^{2}$
This result shows that the variance (equation (33)) is superior to the mean (equation (32)) and the number of visible photons does not follow a Poisson distribution anymore. The difference between the variance and the mean of the distribution is known as Swank noise [32, 33].

The signal to noise ratio can be calculated, for each value of energy, by:

$$
\operatorname{SNR}(E)=\frac{L_{R}(E)}{\sqrt{\sigma_{L R}^{2}(E)}}=\sqrt{N \times T r_{a l} \times A b_{s c}} .
$$

Fig. (13) shows the mean of the number of photons per second, produced by the scintillator, for the input signal of Fig. (9).

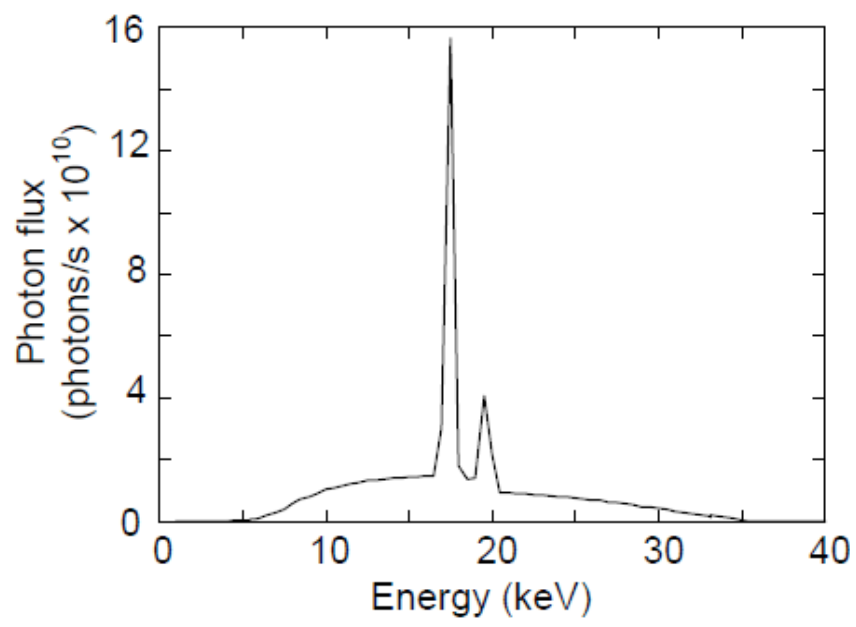

Fig. (13). Number of visible photons produced per second in the scintillator as a function of the incident X-ray energy.

Fig. (14). shows the corresponding signal to noise ratio, obtained from equation (34).

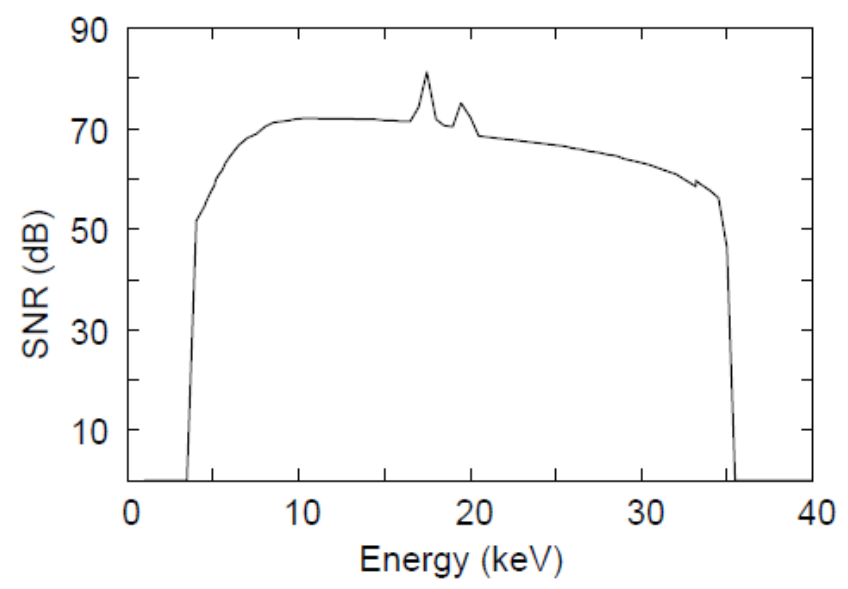

Fig. (14). Signal to noise ratio associated to the visible photons produced by the scintillator.

By comparing the signal to noise ratio of the scintillator Fig. (14) with the one of the X-ray tube Fig. (10), it can be observed that this is practically constant. 
With this result, the first step of the working principle of the X-ray detector based in scintillators is completely characterized. Walter et al. described the applications of an $\mathrm{X}$-ray detector in 2010 [34].

\subsection{Reflection of Visible Light by the Reflective Layers}

The visible light that reaches the reflector can have two independent polarizations. The light that reaches the reflector in an angle $\theta$, measured from the normal, can have either the electric field vector or the magnetic field vector parallel to the plane of incidence. In the first case, the polarization is called $\mathrm{p}$ and in the second case, it is called s. In general, the electric field vector has an arbitrary angle with the incidence plane and can be decomposed into two components, one of polarization $\mathrm{p}$ and another one of polarization $\mathrm{s}$.

The refractive index, $n$, is equal to $H / E$, where $H$ and $E$ represent the amplitudes of magnetic and electric fields respectively. In a similar way, a generalized refractive index, $u$, can be defined for each polarization:

$u_{p}=H /(E \cos \theta)=n / \cos \theta$

$u_{S}=H \cos \theta / E=n \cos \theta$.

Notice that the directions of electric fields of the light produced by scintillators are random and can be decomposed into the two polarizations with equal probability.

In the case of a homogeneous metallic reflector, the reflectivity of the interface between scintillator and reflector is given by:

$R=\left|\frac{u_{s c}-u_{r}}{u_{s c}+u_{r}}\right|^{2}$,

where $u_{s c}$ and $u_{r}$ represent the generalized refractive indexes of the scintillator and the reflector respectively. In the case of materials that absorb light, the value of the refractive index, $n$ of equation (35), has an imaginary component and must be substituted by $n-j k$, where $k$ is the extinction coefficient of the material and $j$ is the complex operator $(\sqrt{-1})$.

In a more generic case of a reflector with multiple interfaces (for example, when a thin film of silicon dioxide is placed between the scintillator and a silicon wall) [35 - 38], the reflectivity can be calculated from [39]:

$\left[\begin{array}{c}E_{q}^{+} \\ E_{q}^{-}\end{array}\right]=\frac{1}{2}\left[\begin{array}{cc}1 & 1 / u_{s c} \\ 1 & -1 / u_{s c}\end{array}\right] M_{i}, \cdots, M_{1}\left[\begin{array}{c}1 \\ u_{w}\end{array}\right] E_{o}^{+}$

where, $E_{q}^{+}, E_{q}^{-}$, and $E_{o}^{+}$represent the amplitudes of the electric field vectors of the incident, reflected and transmitted waves, respectively. Variable $u_{s c}$ and $u_{w}$ represent the generalized refractive indexes of the scintillator and the wall respectively, and:

$M_{i}=\left[\begin{array}{cc}\cos g_{i} & j \sin g_{i} / u_{i} \\ j u_{i} \sin g_{i} & \cos g_{i}\end{array}\right]$

is a matrix that contains the details of thin film $i$, placed between the scintillator and the wall. Basically, $M_{i}$ relates the values of $E$ and $H$ of one side of the film with the ones of the other side. Here, the generalized refractive index of the film is represented by $u_{i}$ and $g_{i}$ is the phase thickness of the film, given by:

$g_{i}=\frac{2 \pi u_{i} d \cos \theta_{i}}{\lambda}$,

where $d$ is the thickness of the film and $\theta_{i}$ is the angle of incidence of the light, given by the Snell law:

$u_{s c} \sin \theta_{s c}=u_{i} \sin \theta_{i}=u_{w} \sin \theta_{w}$.

In this case, the reflectivity is given by:

$R=|r|^{2}=\left|E_{q}^{-} / E_{q}^{+}\right|^{2}$

In the case of the CsI:Tl scintillator, the light produced has a wavelength around $560 \mathrm{~nm}$ [31]. The dependence between the reflectivity and the angle of incidence of the light, for an aluminum reflector, is shown in Fig. (15).

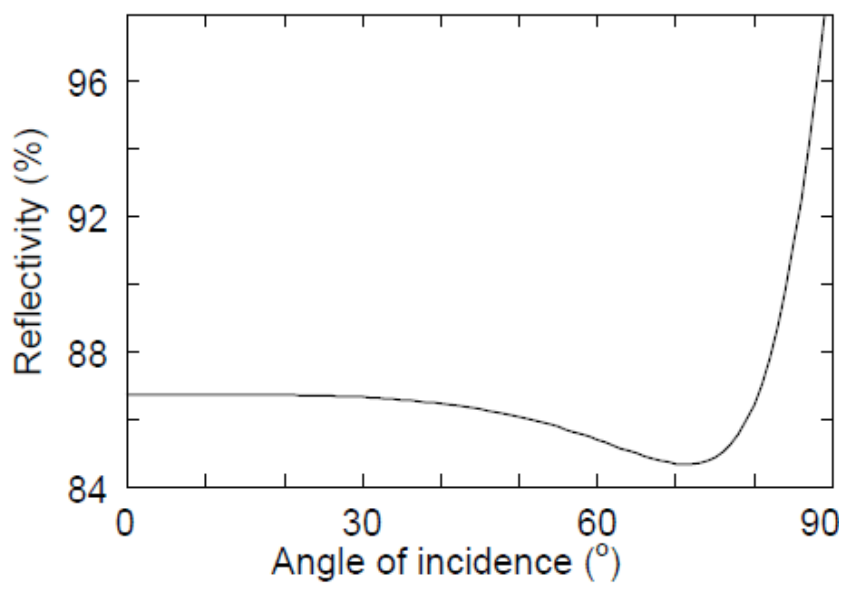

Fig. (15). Dependence between the reflectivity of the visible light and the angle of incidence, for a CsI:Tl - Aluminum interface.

\subsection{Transmission of the Visible Light to the Photodetector}

Due to the differences of refractive indexes between the scintillator and the photodetector, some light produced by the first one is reflected in the surface of the second one. In order to minimize this phenomenon, an anti-reflective filter can be necessary. The simplest way to obtain such filter consists in the application of a thin film in the surface of the photodetector. The parameters of the film can be obtained from equation (42), which was deduced from equation (37):

$$
\begin{aligned}
& E_{s c}^{+}=\frac{1}{2 u_{s c} u_{t f}}\left[\left(u_{s c} u_{t f}+u_{t f} u_{p h}\right) \cos g_{t f}+j\left(u_{s c} u_{p h}+u_{t f}^{2}\right) \sin g_{t f}\right] E_{p h}^{+}, \\
& E_{s c}^{-}=\frac{1}{2 u_{s c} u_{t f}}\left[\left(u_{s c} u_{t f}-u_{t f} u_{p h}\right) \cos g_{t f}+j\left(u_{s c} u_{p h}-u_{t f}^{2}\right) \sin g_{t f}\right] E_{p h}^{+},
\end{aligned}
$$

where $E_{s c}^{+}$is the amplitude of the electric field vector of the incident light (coming from the scintillator), $E_{s c}^{-}$is the amplitude of the electric field vector of the light reflected at 
the interface and $E_{p h}^{+}$is the amplitude of the electric field vector of the light that penetrates into the photodetector. The quantities $u_{s c}, u_{t f}$ and $u_{p h}$ represent the generalized refractive indexes of the scintillator, the thin-film and the photodetector respectively. Finally, $g_{t f}$ is the phase thickness of the thinfilm, given by equation (39).

The reflectivity is given by:

$$
R=\left|\frac{E_{s c}^{-}}{E_{s c}^{+}}\right|^{2}=\left|\frac{\left[\left(u_{s c} u_{t f}-u_{t f} u_{p h}\right) \cos g_{t f}+j\left(u_{s c} u_{p h}-u_{t f}^{2}\right) \sin g_{t f}\right]}{\left.\mid\left(u_{s c} u_{t f}+u_{t f} u_{p h}\right) \cos g_{t f}+j\left(u_{s c} u_{p h}+u_{t f}^{2}\right) \sin g_{t f}\right]}\right|^{2}
$$

For the reflectivity to be null, the numerator of equation (43) must be equal to zero. So, it is enough to make $\cos g_{t f}=0$ and $u_{s c} u_{p h}-u_{t f}^{2}=0$, that is, $g_{t f}=(\pi / 2 \pm k \pi)$, $(k=0,1,2, \cdots)$ and $u_{t f}=\sqrt{u_{s c} u_{p h}}$. A way of verifying both conditions is a thin-film with refractive index equal to $\sqrt{u_{s c} u_{p h}}$ and whose optical path (refractive index times distance) is given by:

$u_{t f} \times d \cos \theta_{t f}=\frac{(2 k+1) \lambda}{4}, \quad k=0,1,2, \cdots$,

that is, a quarter-wave film. Sometimes it is not easy to obtain a material that satisfies the requirement of the refractive index to be equal to $\sqrt{u_{s c} u_{p h}}$. However, for the interface between CsI:Tl scintillator and silicon photodetectors, a good candidate is a film of silicon nitride with $621 \mathrm{~nm}$ of thickness. Fig. (16) shows the values of the reflectivity of the CsI:Tl-Si interface, as a function of the incidence angle, with and without the silicon nitride film. Other good candidates to antireflective films for this application are $\mathrm{ZrO}_{2}$ and the $\mathrm{TiO}_{2}$, with refractive indices of 2.22 and 2.94 , respectively.

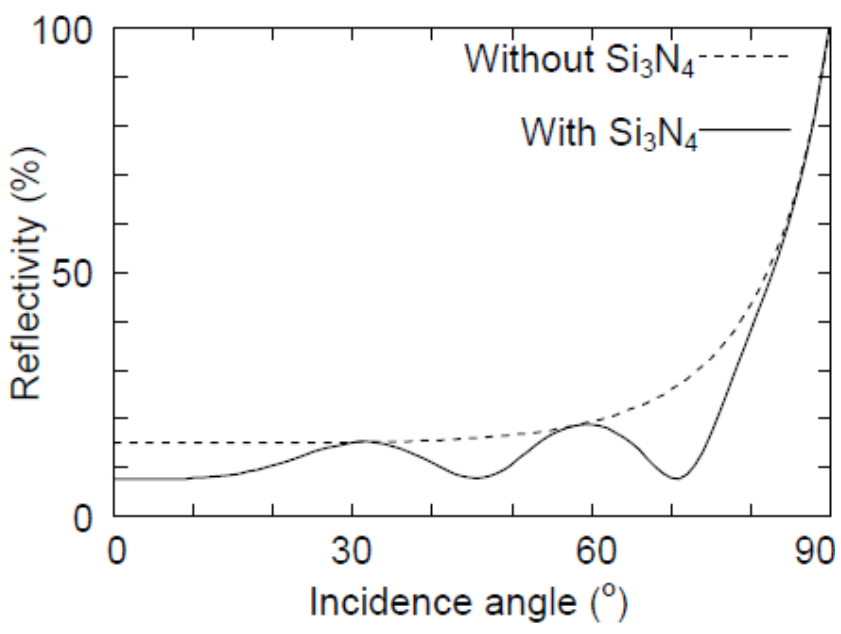

Fig. (16). Dependence between the reflectivity and the angle of incidence of the light, for the CsI:Tl-Si interface, with and without a film of silicon nitride $621 \mathrm{~nm}$ thick.

\subsection{Light Guidance to the Photodetector}

The light produced by the scintillator, after multiple reflections, reaches the photodetector. However, each reflection introduces losses that depend mainly on the geometry of the optical system.

Let's consider a scintillator of prismatic or parallelepipedic form, placed above a photodetector. On the top and in the lateral sides of the scintillator, aluminum layers are placed, which work as reflectors, as it is represented in Fig. (17)

The relationship between the area of the surface of the scintillator seen by the photodetector, $a$, and the total area of its surface, $A$, is given by:

$R_{A}=\frac{a}{A}=\frac{l c}{2 l c+2 h c+2 l h}$

where $l, c$ and $h$ are defined in Fig. (17). Considering that the scintillator is transparent and emits light in all directions with equal probability, the amount of light that reaches directly the photodetector, without any reflection, is given by:

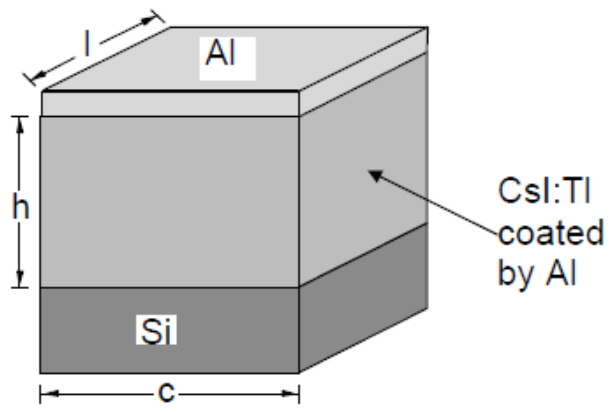

Fig. (17). Scintillator placed above a photodetector, with all its faces coated by reflectors. This configuration represents one pixel of Fig. (8).

$L_{p d}^{*}=L_{R} R_{A}$

where $L_{R}$ is the total light produced by the scintillator. As some light is reflected in the surface of the photodetector, the absorbed one is given by:

$L_{p d}^{* *}=L_{p d}^{*}\left(1-R_{p h}\right)=L_{R} R_{A}\left(1-R_{p h}\right)$,

where $R_{p h}$ is the reflectivity of the surface of the photodetector, given by equation (43). In a similar way, the light that reaches the reflector is given by:

$L_{c m}=L_{R}-L_{p d}^{* *}=L_{R}\left[1-R_{A}\left(1-R_{p h}\right)\right]$.

In the scintillator-reflector interface, an amount of light is lost. The remaining one is reflected back to the scintillator. The percentage of light lost in each reflection is given by:

$R_{\text {loss }}=1-R$

where $R$ is the reflected light, given by equations (36) or (41).

After many reflections, the light that reaches the photodetector is given by: 


$$
L_{p d}=\sum_{i=0}^{\infty} L_{R} R_{A}\left(1-R_{p h}\right)\left[\left(1-R_{A}\left(1-R_{p h}\right)\right)\left(1-R_{\text {loss }}\right)\right]^{i}
$$

Equation (50) represents a geometric series, whose common ratio $\left(1-R_{p h}\right)\left[\left(1-R_{A}\left(1-R_{p h}\right)\right)\left(1-R_{\text {loss }}\right)\right]$ is less than 1 , therefore the series is convergent. So, the light percentage that reaches the photodetector is given by:

$\frac{L_{p d}}{L_{R}}=\frac{R_{A}\left(1-R_{p h}\right)}{1-\left(1-R_{A}\left(1-R_{p h}\right)\right)\left(1-R_{\text {loss }}\right)}$.

Fig. (18) shows the efficiency of the optical system, given by the equation (51), for $c=l=$ pixel size, $h=500 \mu \mathrm{m}$, and without antireflective filter of silicon nitride.

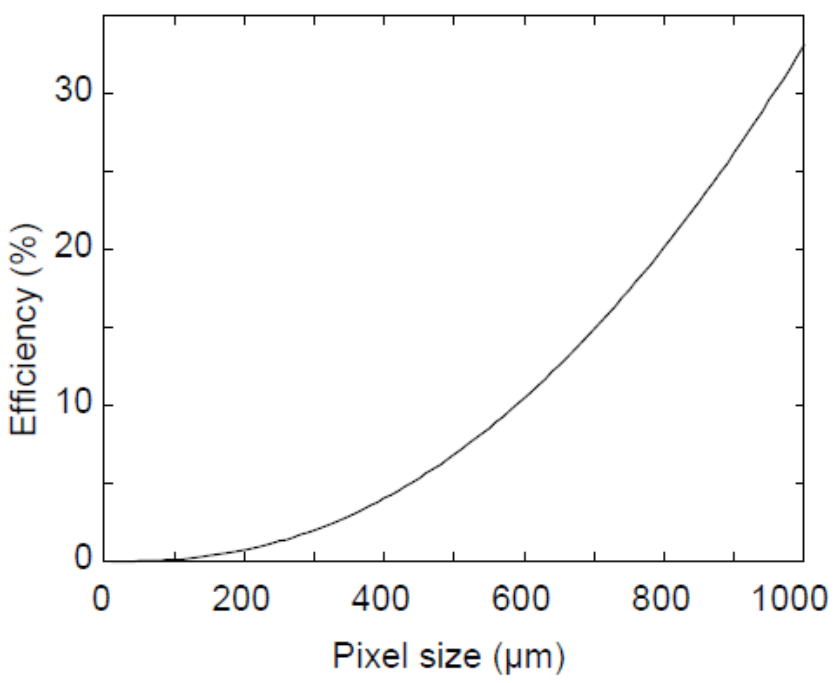

Fig. (18). Efficiency of the optical system for $c=l=$ pixel size, $h=$ $500 \mu \mathrm{m}$, and without silicon nitride antireflective filter.

Detection of visible light by the photodetector.

Fig. (19) shows the quantum efficiency of a CMOS photodetector, manufactured from a $\mathrm{n}+$ /p-epilayer junction, coated by a $\mathrm{Si}_{3} \mathrm{~N}_{4}$ layer. The efficiency at the wavelength of emission of CsI:Tl is marked by dashed lines.

In the case of incident photons following a Poisson distribution, the losses introduced in the system modifies the mean and the variance of the distribution in a similar way, that is, the distribution of electron-hole pairs created in the photodetector is still a Poisson distribution. However, the light produced by the scintillator, due to the randomness of the processes of X-rays production and X-ray conversion into visible light, does not follow a Poisson distribution. In can be described by a normal distribution with mean $m_{s c}$ and variance $\sigma_{s c}^{2}$. In this case, the process of attenuation described by equation (51) can be modeled by a binomial distribution, whose probability of success (the photon is detected) is given by:

$p=\frac{L_{p d}}{L_{R}}$.

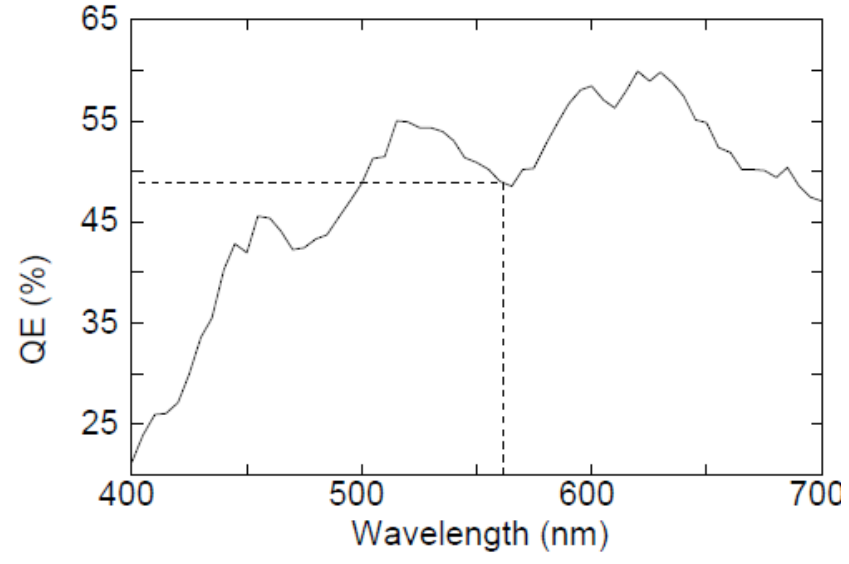

Fig. (19). Quantum efficiency as a function of the wavelength of the incident light, for a $400 \mu \mathrm{m} \times 400 \mu \mathrm{m}$ CMOS photodiode, based in a $\mathrm{n}+/ \mathrm{p}$-epilayer junction and coated by a layer of $\mathrm{Si}_{3} \mathrm{~N}_{4}$.

The distribution of electron-hole pairs generated in the photodetector is obtained by multiplying the distribution of the light produced in the scintillator by this binomial distribution. The mean of the resultant distribution is given by:

$m_{e h p}=p \times m_{s c}$

and the variance is given by [40]:

$\sigma_{e h p}^{2}=p(1-p) m_{s c}+p^{2} \sigma_{s c}^{2}$.

\subsection{Direct Detection of X-Rays by the Photodetector}

The last fact that must be considered is that some X-ray photons cross completely the scintillator and are absorbed by the photodetector. The most usual way to calculate the signal to noise ratio associated with this absorption is the Monte Carlo method [41]. Nevertheless, following a more formal procedure, this absorption can be calculated using the equations (31) to (34), knowing that by each $1 \mathrm{MeV}$ of energy absorbed by silicon, around 277000 electron-hole pairs are produced [36].

Fig. (20) shows the percentage of X-ray photons directly absorbed by a photodiode with a junction depth of $1 \mu \mathrm{m}$, for a CsI:T1 scintillator thicknesses in the range between $100 \mu \mathrm{m}$ and $900 \mu \mathrm{m}$ and $10 \mu \mathrm{m}$ of aluminum on top of it. As in the case of the scintillation mechanism, to obtain the mean value of electron-hole pairs produced, it is necessary to multiply five factors:

- Number of X-ray photons produced by the tube that reach the detector.

- Transmissivity of the reflector placed in the top of the detector.

- Transmissivity of the scintillator.

- 277000 pairs/MeV.

- $\quad$ Energy of the incident photon. 


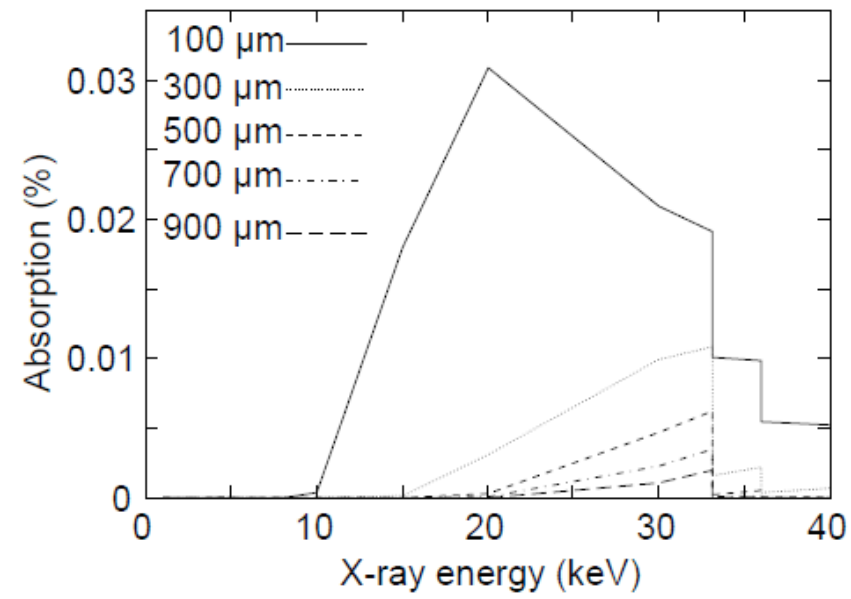

Fig. (20). Percentage of X-ray photons absorbed by a silicon photodiode with $1 \mu \mathrm{m}$ of junction depth, for CsI:Tl scintillators with thicknesses in the range between $100 \mu \mathrm{m}$ and $900 \mu \mathrm{m}$ and a 10 $\mu \mathrm{m}$ layer of aluminum in their top.

Also, as in the case of the scintillation mechanism, the variance of the distribution of electron-hole pairs is given by the product of the mean of X-ray absorbed photons by the square of the number of electron-hole pairs generated by each X-ray photon.

Finally, the contributions of the two processes of generation of electron-hole pair in the photodetector must be added. As both processes can be described by normal distributions, the final result can also be represented by a normal distribution, whose mean is the addition of the two means and whose variance is the addition of the two variances.

The study of the main processes involved in the X-ray detectors based in scintillators, namely the transmission of $\mathrm{X}$-rays through the reflective layer, the absorption of the $\mathrm{X}$ rays by the scintillator and its conversion into visible light, the reflection of the visible light in the reflective layer, the transmission of the visible light to the photodetector, the dependence between the efficiency and the geometry of the pixel and the direct absorption of X-rays by the photodetector is thus concluded.

\subsection{Efficiency of the Scintillator With and Without Light Guides}

At the end of this section, the advantages of the light guides in this kind of detector will be presented. The goal of this section is to prove that, beyond an improvement in the spatial resolution, the introduction of light guides increases the visible light detection efficiency of the detector, which allows a reduction of the X-ray dose in medical imaging.

Fig. (21) shows one pixel of an X-ray detector based in scintillators without light guides. In this case, contrarily to the one presented in Fig. (17), the CsI:T1 has a top aluminum layer, but the other four faces are not coated by reflectors.

When an X-ray photon is absorbed by the scintillator, it produces an amount of visible light, represented by $L_{R}$.

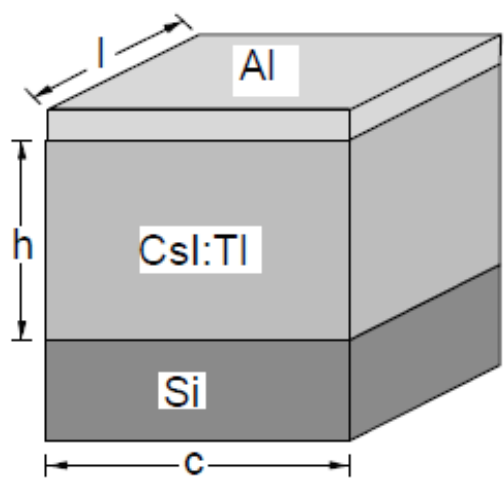

Fig. (21). Scintillator without lateral coatings placed on the top of a photodetector.

The ratio between the scintillator surface area seen by the photodetector, $a$, and its total surface area, $A$, is given by equation (45). Considering that the scintillator is transparent and emits light uniformly in all directions, the amount of light that is directly absorbed by the photodetector, without any reflection, is given by:

$L_{1}^{*}=L_{R} R_{A}\left(1-R_{p h}\right)$,

In the following calculations it is considered that all the points of the aluminum reflector are at the same distance of the photodetector, that is, the reflector instead of being plane, is a small area in the surface of a sphere, whose radius is $h$. In this case, the light reflected in the surface of the photodetector that reaches the reflector of aluminum is given by:

$L_{M 1}^{*}=L_{R} R_{A} R_{p h} \frac{a}{2 \pi h^{2}}$

Being $R_{\text {loss }}$ the percentage of light lost in the reflector, the light that is reflected back to the photodetector is given by:

$L_{2}^{*}=L_{M 1}^{*}\left(1-R_{\text {loss }}\right) \frac{a}{2 \pi h^{2}}\left(1-R_{p h}\right)$

Once again, a light percentage is reflected in the surface of the photodetector and comes back to the aluminum reflector, which reflects it again and thus successively. The total amount of light absorbed by the photodetector is given by the sum:

$$
L^{*}=L_{1}^{*}+L_{2}^{*}+\cdots+L_{n}^{*}=\sum_{i=0}^{\infty} L_{R} R_{A}\left(1-R_{p d}\right) R_{p d}^{i}\left(1-R_{\text {loss }}\right)^{i}\left(\frac{a}{2 \pi h^{2}}\right)^{2 i}
$$

On the other hand, the light produced by the scintillator can go directly to the reflector of aluminum, where it is reflected to the photodetector and thus successively. In this case, the light that is absorbed by the photodetector is given by:

$L^{* *}=L_{1}^{* *}+L_{2}^{* *}+\cdots+L_{n}^{* *}=\sum_{i=0}^{\infty} L_{R} R_{A}\left(1-R_{p d}\right) R_{p d}^{i}\left(1-R_{\text {loss }}\right)^{i+1}\left(\frac{a}{2 \pi h^{2}}\right)^{2 i+1}$

The total amount of light, $L=L^{*}+L^{* *}$, is obtained by adding the terms of the right hand side of equations (58) and (59). After mathematical handling, it can be written as a function of $L_{R}$ : 


$$
\frac{L}{L_{R}}=\frac{2 \pi h^{2} R_{A}\left(a+2 \pi h^{2}-a R_{\text {loss }}\right)\left(1-R_{p d}\right)}{4 \pi^{2} h^{4}+a^{2}\left(1-R_{\text {loss }}\right) R_{p d}}
$$

As practical example, let's consider that the reflectivity of the surface of the photodetector is $5 \%$, the losses in the reflector are $15 \%, h=100 \mu \mathrm{m}, c=30 \mu \mathrm{m}$ and $l=30 \mu \mathrm{m}$. In this case, equation (60) gives a percentage of absorbed light of $6.27 \%$, whereas equation (51) gives a percentage of absorbed light of $30.57 \%$. This means that with the introduction of the light guides, beyond the obvious increase in spatial resolution of the image, the efficiency of the visible light collection of the detector is also increased. By the other hand, the efficiency of the X-ray absorption and visible light production is slightly reduced due to the dead area introduced by aluminum walls. However, this efficiency reduction, which can be calculated by the ratio between the area of the walls and the total area of the matrix, is usually smaller than the figures presented above.

\section{FABRICATION OF A SCINTILLATOR MATRIX}

The fabrication of a matrix of scintillators similar to the one shown in Fig. (7) can be performed by several methods. The production of the cavities can be achieved using a drilling machine, a laser or Deep Reactive Ion Etching (DRIE). The scintillator can thereafter be placed in their interior by pressure at high or low temperatures. The matrix can also be fabricated using a photolithographic process.

In the following sections, the first process will be described in a summarized form, whereas the last one will be more detailed.

\subsection{Production of the Cavities by Using a Drilling Machine, a Laser or DRIE}

In the case of the drilling machine, an aluminum foil with thickness of the order of $500 \mu \mathrm{m}$ is used, in which holes with diameters between $300 \mu \mathrm{m}$ and $400 \mu \mathrm{m}$ are made, with the help of an $x-y$ table. Instead of the drilling machine, a laser can be used, allowing in this case holes with diameters of the order of $100 \mu \mathrm{m}$. Alternatively, the matrix can be fabricated using DRIE. Following a conventional photolithographic process, with a photoresist of $10 \mu \mathrm{m}$ thick, the etching of a silicon substrate can be made using an inductively coupled plasma (ICP) [42], originating a matrix of cavities with perfectly vertical walls and dimensions as small as $50 \mu \mathrm{m}$ (Fig. (22)).

\subsection{Fabrication of the Matrix Using a Photolithographic Process}

The fabrication of the matrix by a photolithographic process is based on SU-8, which is a varnish sensible to ultraviolet light (photoresist). At the beginning of the photolithographic process, sacrificial layers of MicroChem OmniCoat ${ }^{\mathrm{TM}}$ and $\mathrm{SU}-8^{\mathrm{TM}}$ are deposited above the matrix of CMOS photodetectors, as it is shown in Fig. (23a). The OmniCoat is another photoresist that is used to remove the SU-8 after its hardening, as it will be shown later. SU-8 was chosen since it allows deep structures, with very small

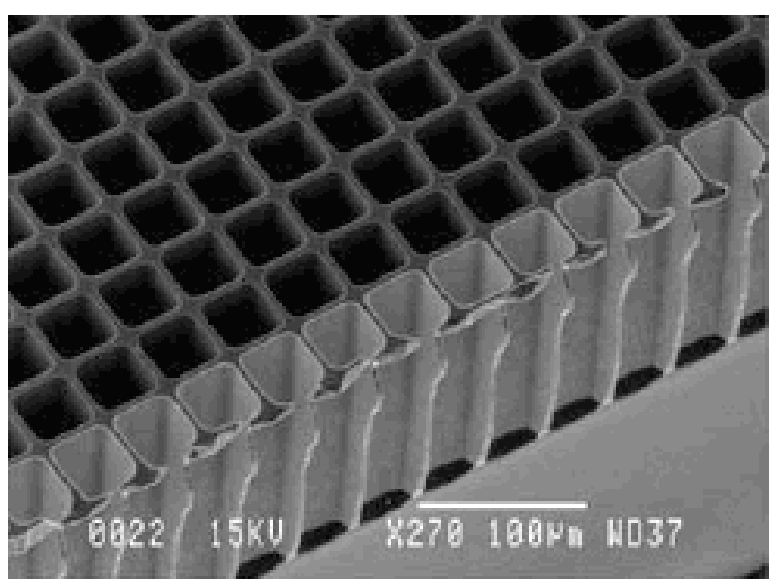

Fig. (22). SEM picture of cavities fabricated by DRIE.

roughness of the walls, which is necessary for the cavities of the light guides with scintillators. Moreover, the fabrication process of SU-8 implies a relatively low cost, when compared with the costs associated with other microfabrication techniques. The necessary steps for fabricating the matrix of scintillators are the following:

- Cleaning of the substrate and placement of OmniCoat.

- Deposition of SU-8.

- Soft bake;

- Placement of mask and exposition to ultraviolet light;

- Post exposure bake;

- Development;

- Washing and drying;

- Placement of aluminum;

- Removal of SU-8;

- Placement of the scintillator inside the cavities;

- Polishing of the top surface;

- Placement of a final aluminum layer in the top of the detector.

Fig. (23) shows these steps, which will be explained with more detail in the following paragraphs.

\subsubsection{Cleaning of the Substrate and Placement of OmniCoat}

In order to obtain the maximum reliability of the process, the substrate must be clean and dry. A bath in a cleaning solvent or a rinsing in a diluted acid, followed by another rinsing in distilled water must be used. After that, the surface must be dried by placing it in a hotplate at $200^{\circ} \mathrm{C}$ during 5 minutes.

Still in this phase, a thin film of OmniCoat will be deposited. The use of this photoresist is essential to remove the SU-8, after the placement of the reflective material of the matrix. The fabrication of this film can be divided into four steps: first, with a spin-coater, about 2 or 3 drops of OmniCoat will be deposited on the substrate. Next, with a spin velocity of $500 \mathrm{rpm}$ during 20 seconds, the OmniCoat 

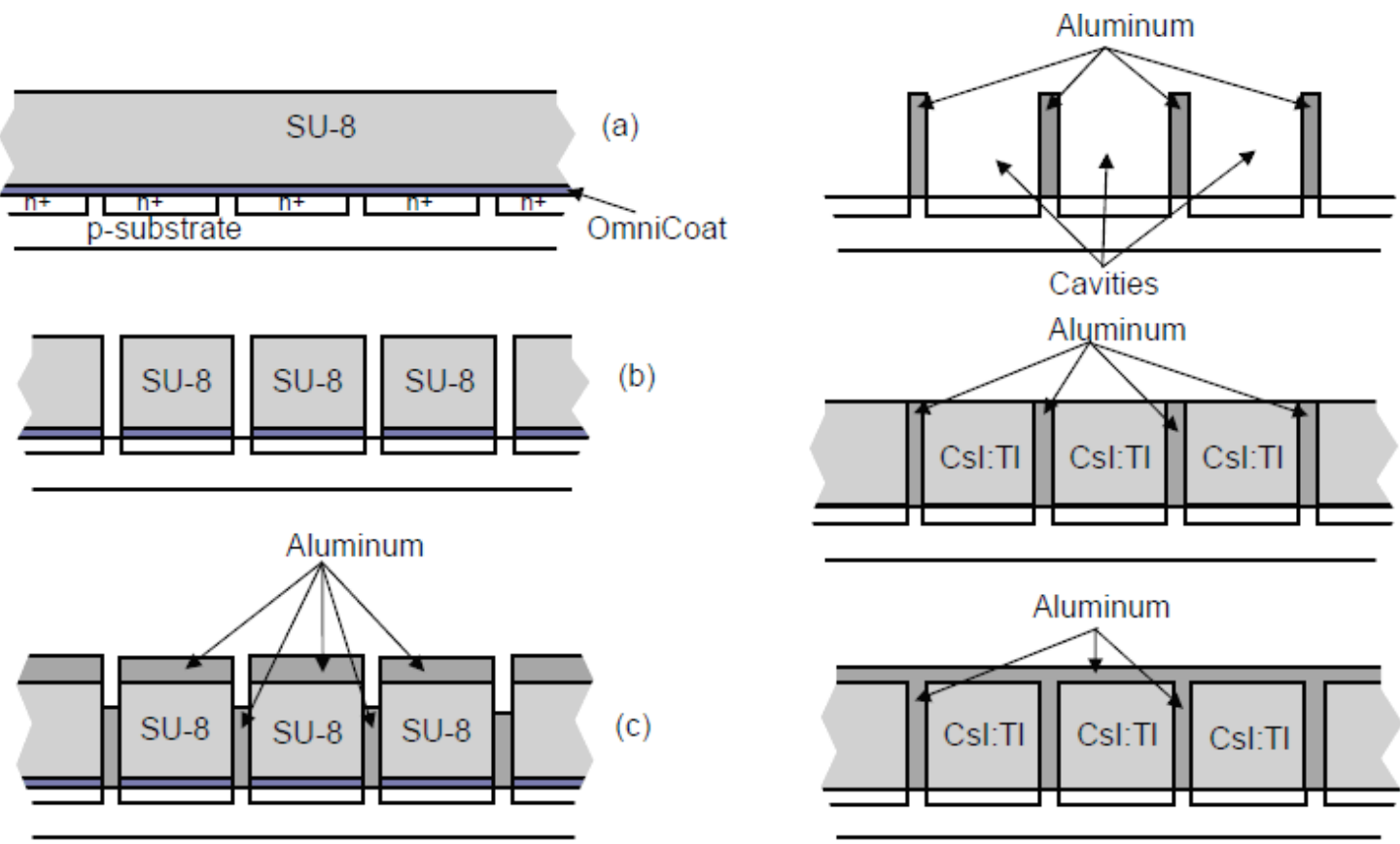

Fig. (23). Fabrication processes of light guides with scintillators: (a) the matrix of photodetectors is coated by OmniCoat and SU-8; (b) after the exposition to ultraviolet light, the parts of SU-8 and OmniCoat not exposed to the light are removed; (c) an aluminum layer is deposited above the matrix; (d) SU-8 and OmniCoat are removed, as well as the aluminum in their top; (e) the scintillator is placed inside the cavities. The upper surface is polished; (f) a final aluminum layer is placed in the top of the detector.

will be spread uniformly. In the third phase, a spin at 3000 rpm during 30 seconds will be performed, in order to obtain a film with thickness in the order of tenths of micrometer. Finally, a heating at $200{ }^{\circ} \mathrm{C}$ for 1 minute will be made in the hotplate.

\subsubsection{Deposition of $S U-8$}

Over the OmniCoat a layer of SU-8 is deposited by spincoating. Fig. (24) shows the relationship between the rotation speed and the obtained thickness, for two types of SU-8 with different viscosities.

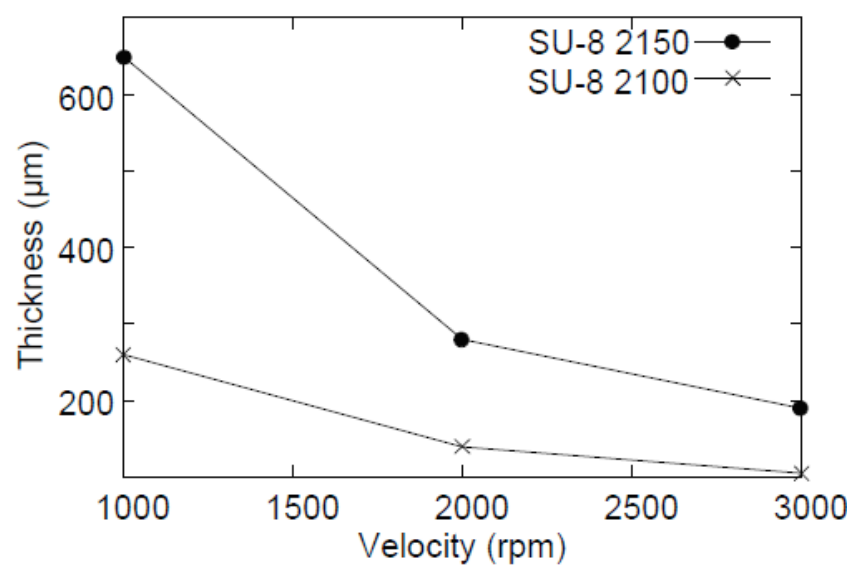

Fig. (24). Obtained thicknesses for two types of SU-8 with different viscosities, as a function of the rotation speed of the spin coater.

After the placement of some amount of SU-8 in the substrate, a first spin at lower speed and a second spin at higher speed will be performed. The first spin has the duration of 20 seconds, at speed of $500 \mathrm{rpm}$, so that the film is spread regularly by the surface of the substrate. The second spin lasts for 30 seconds at the necessary speed to obtain the desired thickness according to Fig. (24).

\subsubsection{Soft Bake}

After having been placed in the substrate, the SU-8 must be heated (soft bake) so that the solvent evaporates and it becomes harder. Soft bake is performed in 2 phases:

1. Heating at temperature $\mathrm{T}_{1}=65^{\circ} \mathrm{C}$, allowing a slower evaporation of the solvent.

2. Heating at the temperature $\mathrm{T}_{2}=95^{\circ} \mathrm{C}$, for evaporating the remaining solvent and hardening the film.

The duration of each phase depends on the thickness of the film. A hotplate with good thermal control and uniformity is recommended for the heating. Conventional ovens are not recommended. Table 1 shows the duration of the soft bake temperatures for SU-8 of series 2000 .

Table 1. Duration of the Soft Bake Temperatures for SU-8 of Series 2000 [43].

\begin{tabular}{|c|c|c|}
\hline $\begin{array}{c}\text { Film Thickness } \\
(\boldsymbol{\mu} \mathbf{m})\end{array}$ & $\begin{array}{c}\text { Duration of } \\
\mathbf{T}_{\mathbf{1}} \text { (Minutes) }\end{array}$ & $\begin{array}{c}\text { Duration of } \\
\mathbf{T}_{\mathbf{2}} \text { (Minutes) }\end{array}$ \\
\hline \hline $100-150$ & 5 & $20-30$ \\
\hline $160-225$ & $5-7$ & $30-45$ \\
\hline $230-270$ & 7 & $45-60$ \\
\hline $280-550$ & $7-10$ & $60-120$ \\
\hline
\end{tabular}




\subsubsection{Placement of Mask and Exposition to Ultraviolet Light}

In this stage of the fabrication process, the SU-8 is exposed to ultraviolet light through an appropriate mask, with the geometry and dimensions of the desired cavities. After a brief cooling, the SU-8 will be exposed to ultraviolet light, using a mask aligner.

SU-8 is optimized to harden with ultraviolet light between $350 \mathrm{~nm}$ and $400 \mathrm{~nm}$. An excessive dose of radiation below $350 \mathrm{~nm}$ results in an exceeding absorption in the surface of the film, hardening it too much. If the ultraviolet lamp has wide spectrum, radiation below $350 \mathrm{~nm}$ must be filtered, in order to obtain better results. The exposure energy that must be supplied to the SU-8 films of series 2000 is in Table 2.

Table 2. Dose of Energy as a Function of the Thickness to be Supplied to SU-8 Films of Series 2000 [43].

\begin{tabular}{|c|c|}
\hline Film Thickness $(\boldsymbol{\mu m})$ & Exposure Energy $(\mathbf{m J . c m}-2)$ \\
\hline \hline $100-150$ & $240-260$ \\
\hline $160-225$ & $260-350$ \\
\hline $230-270$ & $350-370$ \\
\hline $280-550$ & $370-600$ \\
\hline
\end{tabular}

\subsubsection{Post Exposure Bake}

The post exposure bake must be made to selectively bind the parts exposed to the ultraviolet light. Two steps are necessary for this process:

1. Heating at $65^{\circ} \mathrm{C}$.

2. Heating at $95^{\circ} \mathrm{C}$.

According to Table 3, the duration of each step depends on the thickness of the film. After this heating, fast cooling must be avoided.

Table 3. Duration of the temperatures of post exposure bake for the SU-8 of series 2000 [43].

\begin{tabular}{|c|c|c|}
\hline Film Thickness $(\boldsymbol{\mu m})$ & $\begin{array}{c}\text { Duration of } \mathbf{T}= \\
\mathbf{6 5}^{\mathbf{}} \mathbf{C} \text { (Minutes) }\end{array}$ & $\begin{array}{c}\text { Duration of } \mathbf{T}= \\
\mathbf{9 5}^{\circ} \mathbf{C} \text { (Minutes) }\end{array}$ \\
\hline \hline $100-150$ & 5 & $10-12$ \\
\hline $160-225$ & 5 & $12-15$ \\
\hline $230-270$ & 5 & $15-20$ \\
\hline $280-550$ & 5 & $20-30$ \\
\hline
\end{tabular}

\subsubsection{Development}

Before the development of SU-8, a small cooling of the substrate of approximately 15 minutes at room temperature must be performed. Later, the film is immersed in a bath of MicroChem Developer ${ }^{\mathrm{TM}}$, where the parts of the film not exposed to the ultraviolet light, are removed. The time of this process depends on the thickness of the film, as it is shown in Table 4. Agitation of the bath for thicker structures is recommended.

Table 4. Time of Development as a Function of the Thickness for $\mathrm{SU}-8$ of Series 2000 [43]

\begin{tabular}{|c|c|}
\hline Film Thickness $(\boldsymbol{\mu m})$ & Development Time (Minutes) \\
\hline \hline $100-150$ & $10-15$ \\
\hline $160-225$ & $15-17$ \\
\hline $230-270$ & $17-20$ \\
\hline $280-550$ & $20-30$ \\
\hline
\end{tabular}

After this step, the OmniCoat is also developed, using Microposit MF-319 Developer. Columns of SU-8 at the top of the photodetectors are shaped.

\subsubsection{Washing and Drying}

The substrate must be washed with isopropanol and dried with compressed air or nitrogen. If during the washing process, a white film is produced, it means that the development is not complete, and therefore the film must be placed again in the development solution. When this step is finished, the structure of Fig. (23b) is obtained.

\subsubsection{Placement of Aluminum}

The next step is to deposit an aluminum layer in the entire detector surface. The chosen method for this deposition is LIGA (Lithographie Galvanoformung Abformung in German language) [44 - 47]. A thin film (with some tens of nanometers) of aluminum is deposited in the entire matrix by PVD (Physical Vapor Deposition), to form the electrode. Notice that no electric contact is established with the aluminum placed above the SU-8 Fig. (23c), which means that the LIGA process does not deposit aluminum on it.

\subsubsection{Removal of $S U-8$}

MicroChem Remover PG will perform a clean and deep removal of the SU-8, once a film of OmniCoat was applied. The bath must be heated between $50{ }^{\circ} \mathrm{C}$ and $80{ }^{\circ} \mathrm{C}$, and the substrate must be placed in it between 30 and 90 minutes, so that the removal is effective. The thin layer of aluminum that was deposited at the top of SU-8 is also removed, originating the structure of Fig. (23d).

\subsubsection{Placement of the Scintillator Inside the Cavities}

The deposition of the CsI:Tl is a critical process for the implementation of an X-ray image sensor based in this scintillator, once a proper deposition of this scintillator originates X-ray detectors with better spatial resolutions and higher efficiencies, among other advantages. This scintillator is deposited by PVD, but due to the many aspects of the deposition possess, it will be described it in the next section. Shamik et al. explained the versatility of X-ray detector [48].

\subsubsection{Polishing of the Top Surface}

In this step, the CsI:Tl deposited at the top of the aluminum walls will be removed. Its surface will have less roughness too Fig. (23e). 


\subsubsection{Placement of a Final Aluminum Layer in the Top of the Detector}

Finally, a new aluminum layer is deposited by PVD, in the top of the scintillators Fig. (23f).

\subsection{Deposition of Scintillator}

A crucial step of the fabrication of the scintillator matrix consists in the deposition of the scintillator. As it is a relatively complex process with many variables into play, it will be described in this separate section. There are some methods that can be used, namely pressure, melting or PVD. Some of these methods are briefly explained in the following paragraphs.

\subsubsection{Pressure}

In this method, the CsI:Tl scintillator is placed inside the cavities through a pressure of about $10 \mathrm{MPa}$, applied with a machine whose diagram is in Fig. (25) [49].

The scintillator is placed above the cavities. Above the CsI:Tl, a system composed by two plates separated by springs is placed. When the stepper motor rotates, the screw pushes the upper plate, compressing the springs, which in turn makes that the lower plate exerts pressure on the CsI:Tl. From the value of compression of the springs, measured in the scales, their elasticity constant and the area of the surface of CsI:Tl, the exerted pressure is calculated. In order to assure that the cavities are completely filled, the system of Fig. (25) is placed inside of a vacuum chamber, so that the air inside the cavities does not make the process difficult.

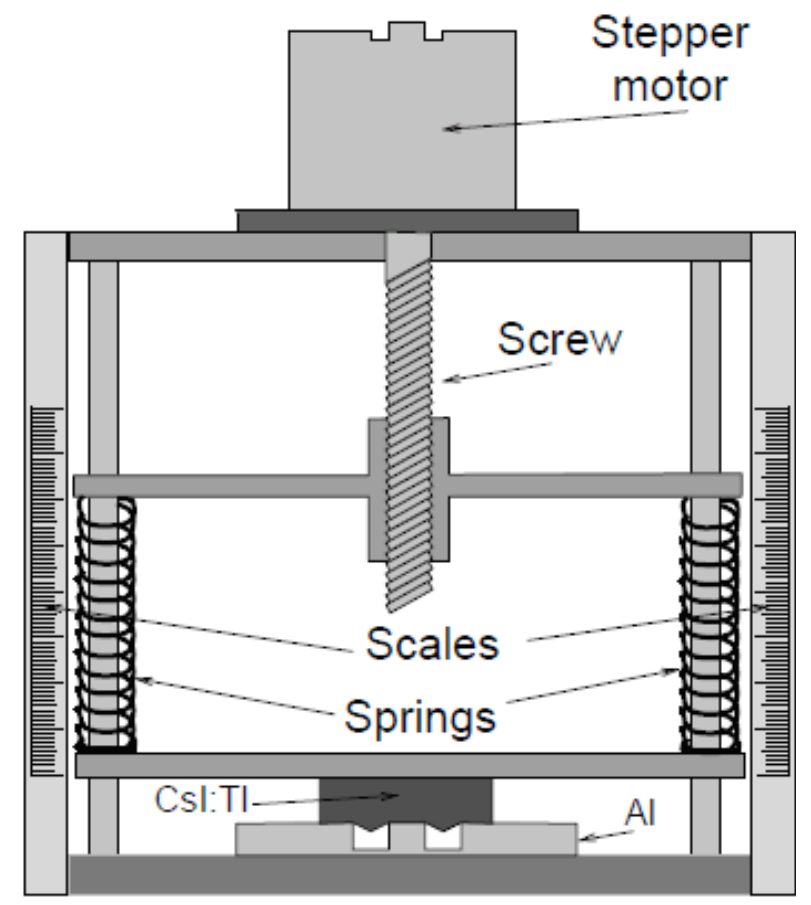

Fig. (25). Schematic diagram of a system for placing the scintillator based on pressure.

After this step, an annealing procedure is necessary to reorganize the crystals.

\subsubsection{Melting}

In this method, the CsI:Tl is melted at $621{ }^{\circ} \mathrm{C}$ in the top of the cavity matrix. The matrix is later submerged in the liquid assuring a complete filling of the sockets.

As it can be seen in Fig. (26), a spring keeps the matrix in thermal contact with the sample-holder during the process, preventing that it floats.

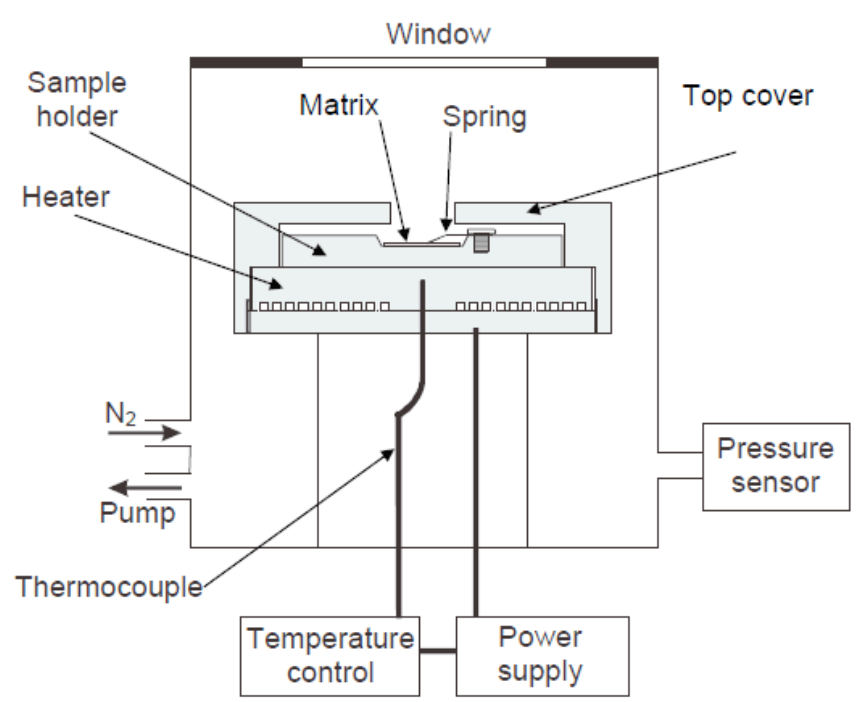

Fig. (26). Schematic diagram of the melting system for placing the CsI:Tl inside the cavities [50].

The system operates in a nitrogen atmosphere, so that oxidation is minimized and thermal conduction by convection is allowed. The electrical power supplied to a heating resistor, which is placed underneath the sample-holder, is controlled by a thermocouple. As final step of this technique, it is necessary to polish the surface to eliminate some CsI:Tl that is above the aluminum.

\subsubsection{Physical Vapor Deposition (PVD)}

It is known that the deposition of the CsI:Tl by physical processes under vacuum conditions improves the performance of the X-ray detectors that use this scintillator, once the deposition of these films in vacuum stimulates the appearance of columnar microstructures and promotes the development of more uniform and homogeneous films [51]. The deposition by PVD that uses resistive heating is the most common process of deposition of this scintillator, since it is a relatively simple and cheap method, it is not aggressive for materials, it has good deposition rates and minimum contamination of the films due to the used high vacuum level. However, the use of vacuum evaporation techniques can be problematic to deposit a film with the same stoichiometric composition of the source material when, as it is the case, the vapor pressure of its constituents are completely different [52]. Moreover, the final composition of the film can be non-homogeneous in the entire thickness of it, due to difference of volatilities of its elements. It is a general rule that the element of higher vapor pressure, in this in case is thallium iodide (TII), will have the higher probability of being vaporized, and thus the thallium will be in higher concentration in the final matrix than initially in the source 
material [52]. In this process it is also important to take into account the dissociation energy. In this particular case, the CsI is a composite thermally very stable, and in such a way its dissociation, in iodine and cesium during the vaporization process, is irrelevant [53].

Usually, the source material to be deposited is obtained by mixing CsI and $\mathrm{Tl}$ powder, with the desired concentrations. In this process, metallic powder of $\mathrm{Tl}$ is preferable instead of TII salt, due to its smaller vapor pressure [54].

A more efficient control of the composition of CsI:Tl can be made with coevaporation, where CsI and the $\mathrm{Tl}$ are vaporized from different sources, with independent control of temperatures. Another alternative solution would be to deposit a film of TII above the film of CsI, followed by an annealing process to spread thallium for the entire film of CsI [51]. The properties of the CsI:Tl film can still be optimized by the correct parameterization of the deposition conditions [55]. The optimal conjugation between temperature and evaporation pressure, properties and temperature of the substrate, deposition rate, thallium concentration and thermal treatments after deposition globally improves the quality of the obtained films.

\section{Evaporation Pressure}

Depositions in conditions of low vacuum and low temperatures of the substrate tend to form microstructures in the form of quadrangular needles much less dense than in conditions of high vacuum.

When the CsI:Tl scintillator is deposited in conditions of high vacuum and low temperature of the substrate, higher light intensities are obtained, when the film is subject to the radiation [54]. High temperatures of substrate, higher than $100{ }^{\circ} \mathrm{C}$, cause the vaporization of TII, which is more volatile than CsI, forming a film with smaller concentration of thallium [56].

\section{Properties and Temperature of the Substrate}

The crystalline orientation of the substrate conditions the orientation of the CsI:Tl film. In the case of a substrate with certain orientation, it is originated a film of CsI:Tl with a perfect columnar structure, because some orientations are preferential, usually different of the crystalline orientation of the substrate [51]. Amorphous substrates foment the formation of polycrystalline structures [55]. In these films, the presence of grain borders promotes the dispersion and the absorption of the produced light, making the light propagation through the columns more difficult [51].

With higher substrate temperatures, the deposition results in fibers of higher sizes, very dense and with better adhesion the smooth surfaces. With lower substrate temperatures, thinner columnar structures were obtained $[54,57]$. In the conditions of low temperatures, the substrate must have a rougher surface in order to improve the adhesion [57].

\section{Deposition Rate}

It is confirmed that slower evaporation rates tend to originate more uniform CsI:Tl films and with higher thallium concentrations $[51,55]$, resulting in a higher intensity of produced light. Fig. (27) shows the relationship between the

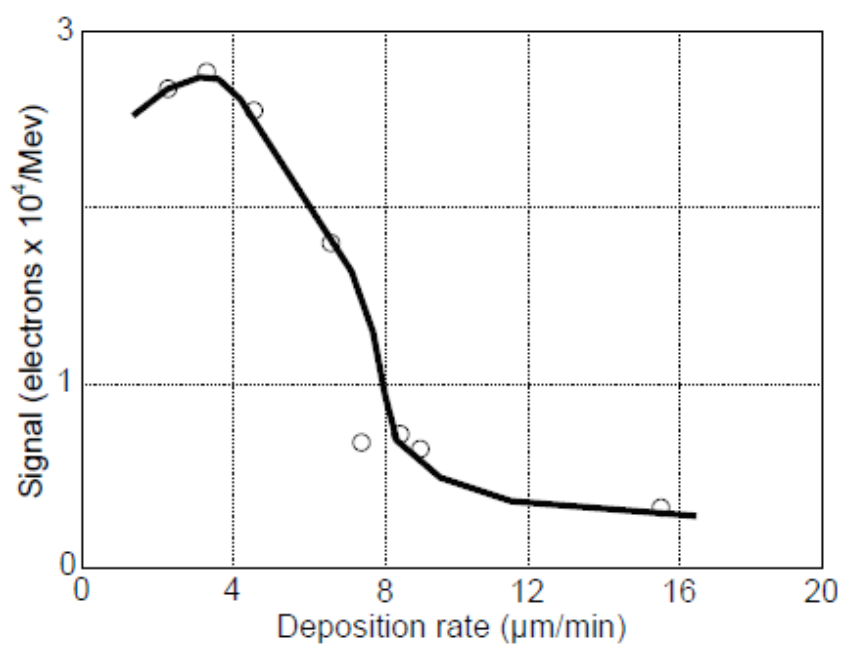

Fig. (27). Relationship between the deposition rate and the output signal of a photodetector, obtained with an X-ray source of $50 \mathrm{kVp}$ [57].

deposition rate of CsI:Tl and the production of visible light by the resulting crystal when radiated by a $50 \mathrm{kVp}$ X-ray source.

\section{Thallium Concentration}

Different thallium concentrations promote certain crystalline orientations [54]. Moreover, the intensity of light produced by the CsI:Tl scintillator is highly sensible to the thallium concentration, since the thallium atoms work as activators in alkali-halide scintillators, and therefore they promote the efficiency in the light production, as well as the production of light with higher wavelengths. A homogeneous thallium concentration is necessary to optimize the performance of the CsI:Tl scintillator [58]. Fig. (28) shows the relationship between some properties of the CsI:Tl and the thallium concentration.

\section{Thermal Treatments After Deposition}

A thermal treatment of annealing after the deposition of the film of CsI:Tl improves its optical properties. This treatment promotes the thermal diffusion of atoms of $\mathrm{Tl}$ and CsI, originating the creation of more luminescence centers. In this process, the increase of temperature causes an increase of the light production, however high temperatures (superior to $500{ }^{\circ} \mathrm{C}$ ) induces degradation the light production, once the atoms of $\mathrm{Tl}$ evaporate [52]. Table $\mathbf{5}$ shows the production of light of some CsI:Tl samples before and after thermal treatments of annealing.

\section{PHOTODETECTORS AND CMOS INTERFACE ELECTRONICS}

In the previous sections theoretical studies about the production and detection of X-rays, scintillators and optical interfaces have been carried through. In order to complete the study of the X-ray microdetectors based on scintillators, this section is dedicated to the photodetectors and the interface electronics. 


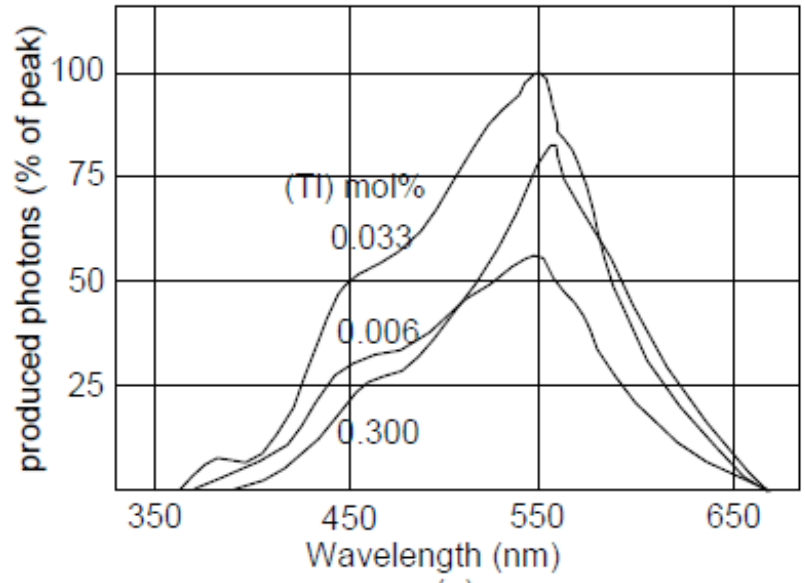

(a)

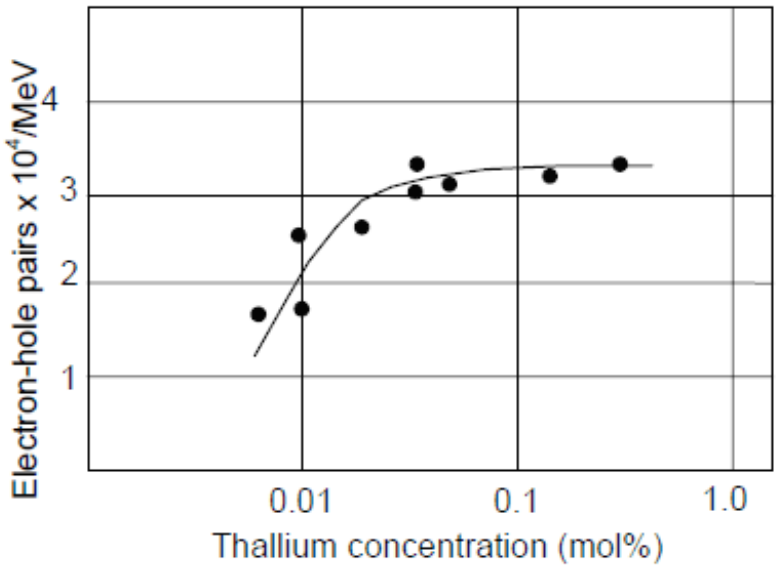

(b)

Fig. (28). Optical properties of CsI:Tl as a function of the thallium concentration [59]. (a) Emission spectra; (b) Production of light, measured in a silicon photodiode, for gamma rays of $622 \mathrm{keV}$.

Table 5. Production of Light by CsI:Tl Crystals Before and After a Thermal Treatment of Annealing. Conditions of Deposition: A - CsI:TI $0.3 \mathrm{~mol} \%$, Temperature of the Substrate of $35^{\circ} \mathrm{C}$, Pressure of $10^{-5}$ torr; B - CsI:Tl $0.3 \mathrm{~mol} \%$, Temperature of the Substrate of $30^{\circ} \mathrm{C}$, Pressure of $10^{-5}$ torr; C - CsI:Tl $0.3 \mathrm{~mol} \%$, Temperature of the Substrate of $250^{\circ} \mathrm{C}$, Pressure of $10^{-5}$ torr. The Results Were Obtained with an X-ray Source of 80 kVp [54].

\begin{tabular}{|c|c|c|}
\hline & \multicolumn{2}{|c|}{ Produced Light (\% of Maximum) } \\
\hline Deposition Conditions & Before Annealing & After Annealing \\
\hline \hline A & 97 & 100 \\
\hline B & 86 & 92 \\
\hline C & 50 & 57 \\
\hline
\end{tabular}

\subsection{Photodetectors in CMOS}

CMOS fabrication process is adjusted for digital electronics designs allowing however to manufacture analog electronics and some kinds of photodetectors [60]:

- Vertical photodiodes (three different types).

- Lateral photodiode.

- Vertical bipolar phototransistor.

- Lateral bipolar phototransistor.

- Photogate.

- Avalanche photodiode [61]. them.

The schematic diagram of Fig. (29) shows each one of

\subsubsection{Vertical Photodiodes}

The working principle of the three types of vertical photodiodes Fig. (29a, b c) is identical. Between them only the junction depth and the concentration of impurities is different. All of them use the photoelectric effect [62] to convert photons into electron-hole pairs. When a photon with higher energy than the bandgap of silicon $(1.124 \mathrm{eV})$ is absorbed, it excites an electron to the conduction band, leaving a hole in the valence band. This means that all photons with wavelength smaller than $1.1 \mu \mathrm{m}$ have some probability to excite electrons from the valence band to the conduction band. These electrons, as well as the holes of the valence band, move freely through the crystal lattice, originating electric currents. This means that, using to advantage the photoelectric effect, transducers that convert the incident light into electrical current can be manufactured. The electron-hole pairs will form an electric current if their recombination is avoided.

One of the simplest methods to separate the electron-hole pairs and prevent their recombination consists of using an inversely polarized pn junction, which originates a region free of charges (depletion region), in which the electric field is very intense. If an electron-hole pair is generated in the depletion region (or close to the depletion region and penetrates there by diffusion), the electric field quickly separates the electron from the hole. The electron travels to the $\mathrm{p}$ side and the hole to the $\mathrm{n}$ side, originating an electric current through the junction. This current is proportional to the intensity of the incident light.

The absorption of visible light by a semiconductor depends on its wavelength. The radiation of short wavelength, that is, of higher energy is quickly absorbed at small depth. In the opposite, the radiation of longer wavelengths penetrates much deeply before being absorbed. Notice that this behavior is valid only for the visible region of the spectrum (or close to it). In other regions of the spectrum, as in the X-ray range, the behavior of photons is very different. The absorption length, defined as the distance in which $1 / \mathrm{e}(\mathrm{e}$ $\simeq 2.71828$ ) of incident photons are absorbed, is close to 0.3 $\mu \mathrm{m}$ for the blue light of $475 \mathrm{~nm}$ and $3 \mu \mathrm{m}$ for the red light of $650 \mathrm{~nm}$ [63]. This behavior is explained by the distribution of probability of photons with some energy to excite carriers of the valence band to the conduction band. Visible light photons of higher energy have higher probability to excite electrons of the valence band, then they are absorbed more 


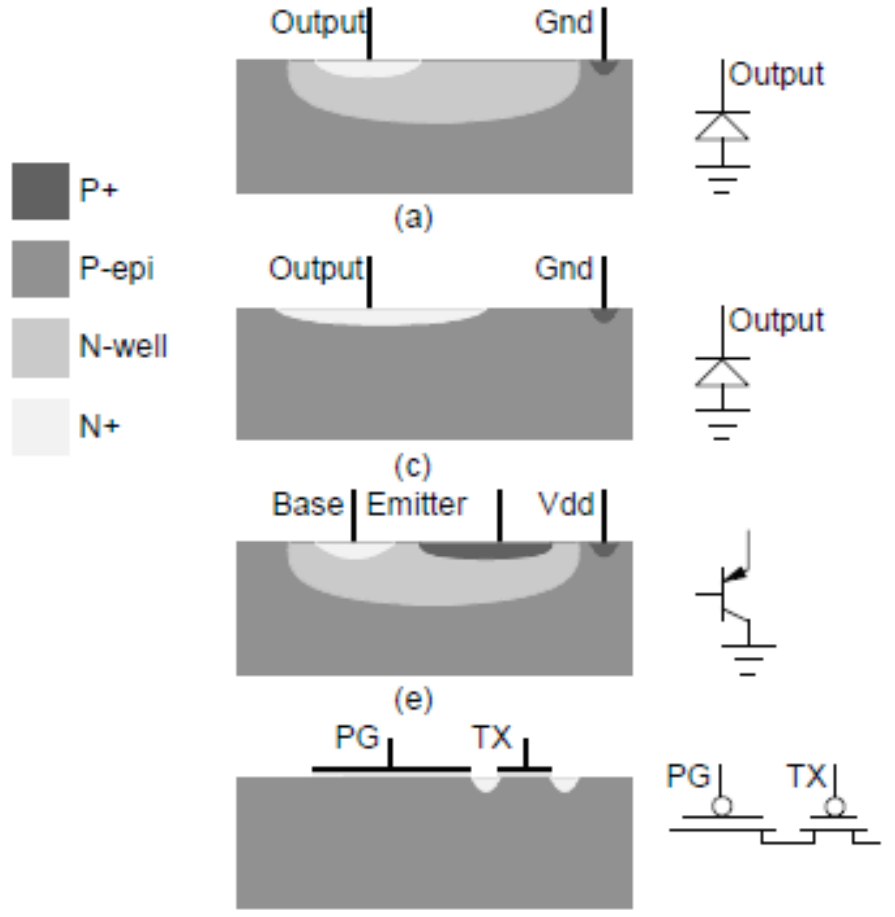

(g)

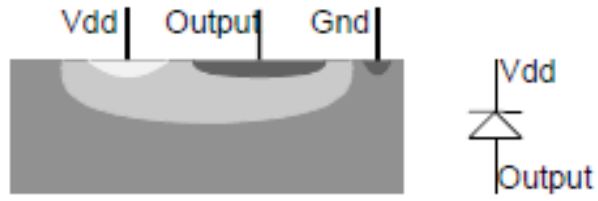

(b)

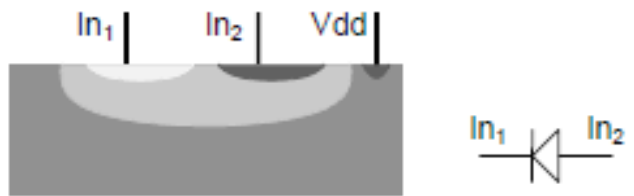

(d)

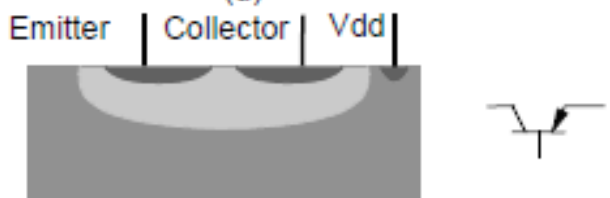

(f)

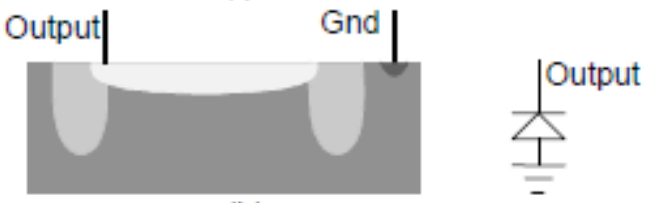

(h)

Fig. (29). CMOS photodetector structures. (a) n-well/p-epilayer vertical diode. (b) p+/n-well vertical diode. (c) n+/p-epilayer vertical diode. (d) Lateral diode. (e) Vertical bipolar transistor. (f) Lateral bipolar transistor. (g) Photogate. (h) Avalanche photodiode.

quickly. The dependence between the absorption and the wavelength justifies the different behavior of the three types of photodiodes. The junction n-well/p-epilayer is the deepest of the three, so this photodiode has a better response to longer visible wavelengths. The junction $\mathrm{p}+\mathrm{n}$-well is close to the surface, therefore this photodiode has a better response to shorter wavelengths. The junction $\mathrm{n}+/ \mathrm{p}$-epilayer has an intermediate depth, so the response of this photodiode to different wavelengths is also intermediate relatively to the other two.

\subsubsection{Vertical Phototransistor}

Vertical phototransistors Fig. (29e) also use the photoelectric effect to convert photons into electron-hole pairs. The electron-hole pairs, generated by the photoelectric effect, are separated and collected using a pn junction inversely biased, very close to another pn junction directly biased.

As in a photodiode, the incident radiation in the inversely biased junction (collector/base) causes an electric current that is proportional to the intensity of the light. This current flows into the region of the base, which means that it is multiplied by the gain parameter $\beta$ of the transistor. As the value of $\beta$ is typically of the order of the tens or hundreds, after this gain multiplication, the phototransistor can show quantum efficiency higher than 1 in the visible light spectrum.

\subsubsection{Lateral Devices, Photogates and Avalanche Photodiodes}

The working principle of lateral diodes and bipolar transistors Fig. (29d) and (f) respectively) are similar to the corresponding vertical ones. The main difference is in the spectral response. The structures of lateral diodes and bipolar transistors have a good response in the blue region, due to the fact that in these types of photodetectors, the junction extends to the surface, where most of the blue light is absorbed.

Photogate Fig. (29g) consists in a capacitor between the gate (terminal PG in Fig. (29g) and the substrate. When a voltage is applied to the gate, a depletion region in the zone below it is created. The electron-hole pairs generated in that zone are separated and contribute for the current of the photogate [61].

Of all the structures, this is the one that has smaller quantum efficiency due to the fact that the generated current is mainly due to carrier diffusion and because the material used in the gate absorbs most of photons of small and medium wavelength [60]. In order to reduce this second problem, the gate material must transmit the maximum possible number of photons in the wavelengths of interest. Preferably, silicon composites or metals must not be used $[64,65]$. To increase the light transmission, in the case of the photodetector is big enough, small windows can be opened in the gate.

The avalanche photodiode Fig. (29h) can be implemented from a $\mathrm{n}+/ \mathrm{p}$-epilayer junction and a ring shaped from a $\mathrm{n}$ well region. This ring is used to prevent the premature deterioration of the diode from its lateral borders, due to the fact that it is inversely biased close to the rupture zone. Normally, this diode is biased with voltages of several tens of volts, and when it receives light, it occurs an effect similar to the avalanche that happens in zenner diodes. 


\subsection{Pixel Structures}

When X-ray detectors are developed for obtaining images, it is necessary to use the photodetectors in matrix form, together with some interface electronics. The set photodetector - electronics forms the pixels of the detector.

There are three basic pixel structures in CMOS image detectors: the passive pixel sensor (PPS), which was the first one to be developed, the active pixel sensor (APS), which was developed with the goal of improving the quality of image, and the digital pixel sensor (DPS). The APS has an amplifier stage, whereas the PPS has only one commutation transistor. The DPS is characterized by having an analog to digital converter for each pixel. Martin et al. disclosed a large-area CMOS flat X-ray detector. The detector includes a plurality of CMOS detector chips [66].

\subsubsection{Passive Pixel Sensor}

The PPS [67, 68] was the first CMOS image sensor available in the market, but its development was discontinued due to factors related with the signal to noise ratio (SNR). Each pixel of a PPS consists basically in a photodiode and a commutation transistor, as it is shown in Fig. (30). The readout of pixels is processed in a similar way as a DRAM memory.

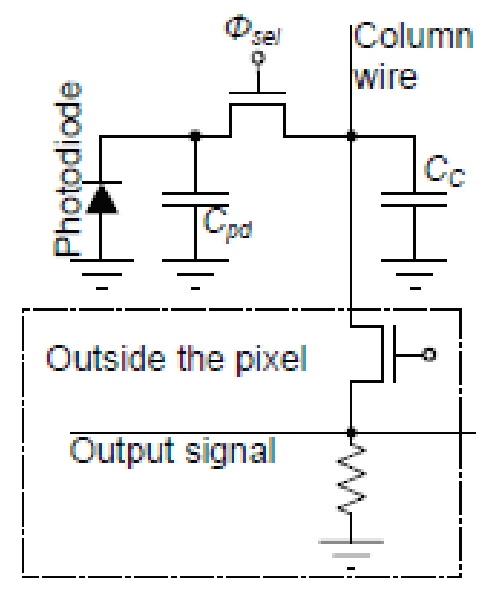

Fig. (30). Basic circuit of a PPS. Capacitor $C_{p d}$ represents the capacitance of the pn junction and capacitor $C_{C}$ is the capacitance of the column wire.

Due to its simple structure, PPS has high fill-factor (ratio between the photosensitive area and the total area of each pixel), but the commutation noise is a very negative factor. In the PPS circuit of Fig. (30), the accumulated charge is read through a resistor placed outside of the matrix. Another process would be to use a charge amplifier instead of the resistor $[67,68]$.

This configuration has some disadvantages, namely:

- High smear noise.

- High reset noise $(k T / C)$.

- High fixed pattern noise.
Due to these disadvantages, the transversal signal line passive pixel sensor (TSL-PPS) was developed [69 - 71], where a column selection transistor is placed in each pixel Fig. (31).

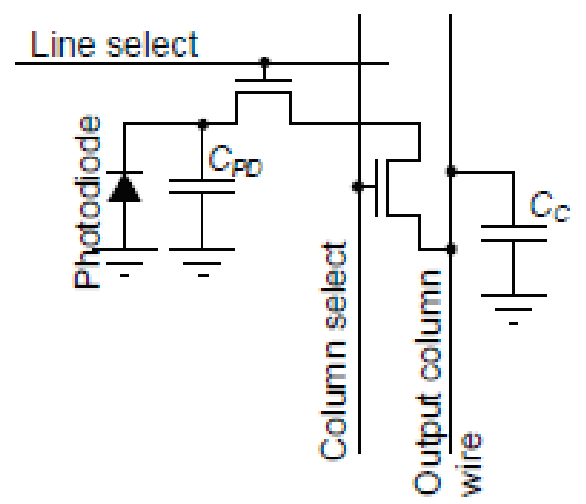

Fig. (31). Basic structure of a TSL-PPS [69].

In opposite to the basic PPS, the TSL-PPS allows to disconnect all pixels, except the one that is being read. With this procedure, the smear noise is eliminated [72].

\subsubsection{Active Pixel Sensor}

The APS is characterized by having an active element that amplifies the signal of each pixel. Fig. (32) shows the APS configuration with three transistors (3T-APS), where, besides the line and column selection transistors, there is a third one, connected in a common drain amplifier configuration, which improves the signal to noise ratio of the image. Unhappily, $k T / C$ noise is still present.

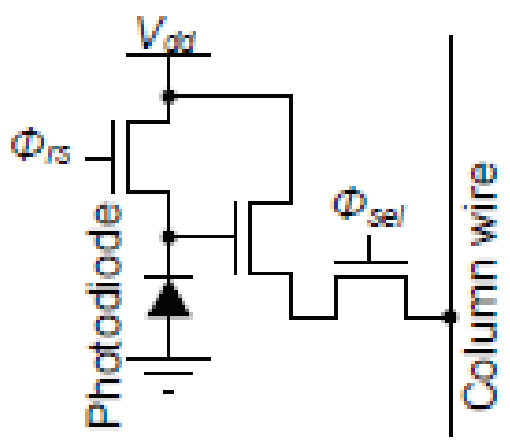

Fig. (32). Basic structure of a 3T-APS.

\subsubsection{Digital Pixel Sensor}

In the pixel structures previously presented, the analog to digital conversion is performed outside of the matrix. In the DPS, each pixel contains the photodetector, the analog to digital converter and sometimes, the memory necessary to keep the value of the conversion. Fig. (33) shows its basic structure.

In this way, a truly parallel conversion of all pixels and a fast access to the memory of each one is possible. The transmission of digital signals is much more immune to 


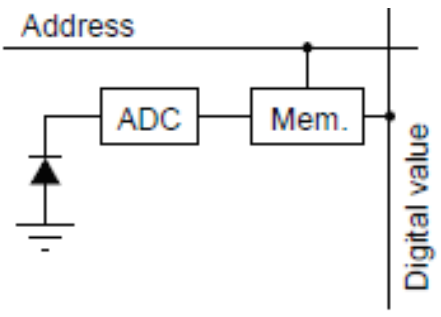

Fig. (33). Basic structure of a DPS.

noise, so high-performance transmission lines are not necessary. The proximity between photodetector and analog to digital converter also eliminates the analog multiplexing circuits and hence the noise introduced by their commutations.

The reduced space for the implementation of the analog to digital converting circuit has stimulated the development of simpler and more programmable structures.

DPS architecture has some advantages relative to analog image sensors, namely a better adaptation to CMOS technology, since it does not need high-performance analog circuits, and it eliminates the readout noise associated with each column [73].

The biggest disadvantage of the DPS is that it needs more transistors per pixel than conventional analog image sensors, therefore it must have bigger pixel sizes with more reduced fill-factors.

As CMOS technology develops, it is becoming possible to integrate more transistors in each pixel, which explains the fact that in the future all image sensors tend to evolve to the DPS type [74]. Happily, the pixel area occupied by the ana$\log$ to digital converter and the consequent reduction of the fill-factor does not occur for the X-ray image sensors based on scintillators and light guides [75], since in this architecture, each scintillator is separated from its neighbors by reflective walls. The analog to digital converter of each pixel can be placed underneath the reflective walls, not decreasing the photodetector area or the fill-factor.

The first DPS converters were based in pulse width modulation (PWM) or pulse frequency modulation (PFM). They are represented in Fig. (34).

The PWM converter works generally in a single slope configuration, using the time of charge of a capacitor and a differential amplifier or a digital inverter as comparator [76].
In the PFM converter, the charge and discharge times of a capacitor defines the frequency of the output pulses [77].

A little more elaborated approach uses a single slope analog to digital converter, constituted by around 40 transistors $[73,74,77,78]$.

DPS structures with sigma-delta converters have also been proposed, with resolutions of 12 bits or more [79 - 83]. According to these and other authors, the sigma-delta modulation allows for conversion circuits that are simple and insensitive to variations of the fabrication process [82,84].

In a general way, as it will be demonstrated in the following sections, the first-order sigma-delta converter has the best performance of all known converters used in DPS devices, having physical dimensions equal or smaller than the others.

\subsection{First-Order Sigma-Delta Modulation}

In a DPS image sensor with the sigma-delta type, each pixel is constituted by a photodetector and an analog to digital converter. The blocks of temporization and control logic are also integrated into the same chip, but outside the pixel matrix. This architecture allows several ways of image readout, where the gradual scanning of the entire matrix is the most common. The readout of some area of interest of the matrix, where only a small region is selected, or the random access to each pixel, are also possible. These readout ways increase the access rates to interest areas, which together with changeable oversampling rates allow increasing of the readout speed, with lower resolutions, for example.

In the design that will be presented throughout this section, the addressing of the columns of the matrix is made using clock signals shifted in time, allowing that the digital outputs of all pixels of a line are connected to the same wire. In this case, if the line has $\mathrm{N}$ pixels, the duty-cycle of the clock signal must be smaller or equal to $(1 / N) \times 100 \%$.

The communication between each pixel and the exterior of the matrix is made by a current signal (and not a voltage one), that is, each time a pixel sends a logic 1 , a current pulse is injected into the output wire.

Fig. (35) shows a block diagram of the circuit of each pixel, which contains, besides the photodetector, a first-order sigma-delta modulator. When an image is focused on the photodetector matrix, the sigma-delta converters start the
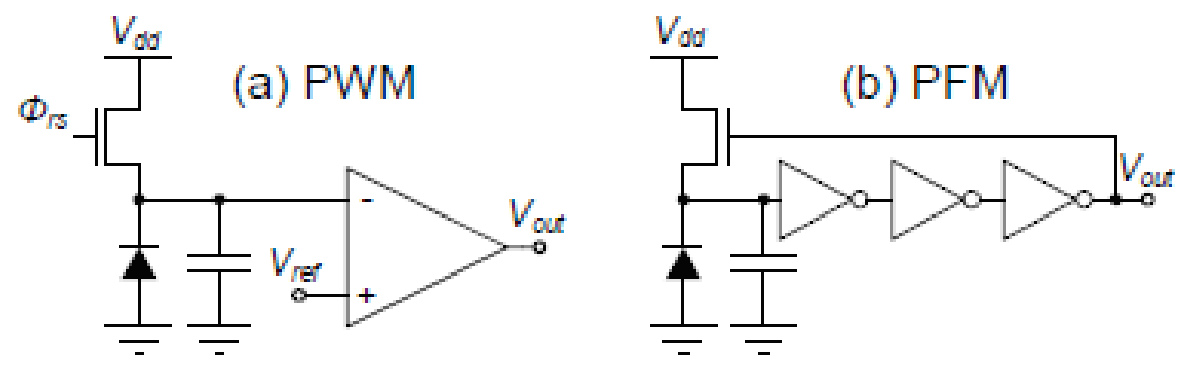

Fig. (34). Basic structures of analog to digital conversion for DPS: (a) Pulse width modulation (PWM); (b) Pulse frequency modulation (PFM). 


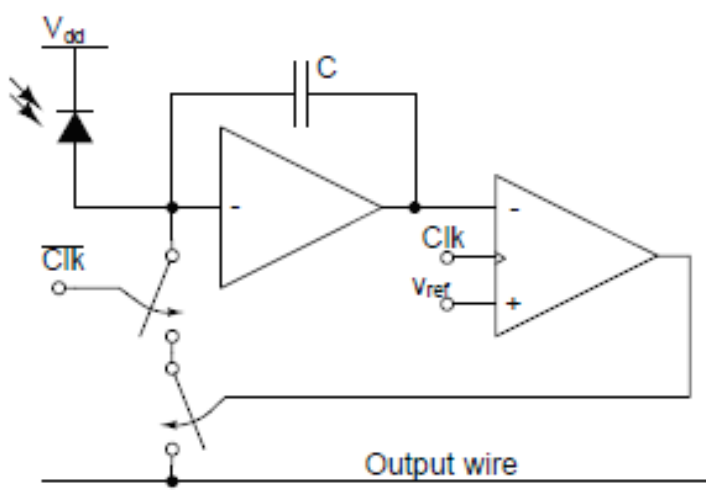

Fig. (35). Block of each pixel.

conversion. The oversampling ratio is defined by the desired resolution, that is, the number of output bits. This means that the oversampling principle allows a flexible compromise between the conversion time and the precision of the result.

In the particular application of the X-ray image sensor, a 1 bit first-order sigma-delta converter, with an oversampling ratio of 256 or 512, can be used, with a not very high clock frequency (1 MHz, for example). The digital values that come from each sigma-delta converter are reconstructed using a decimation filter, which can be implemented in the hardware or programmed in a microcontroller.

Fig. (36) shows the complete schematic diagram of each pixel.

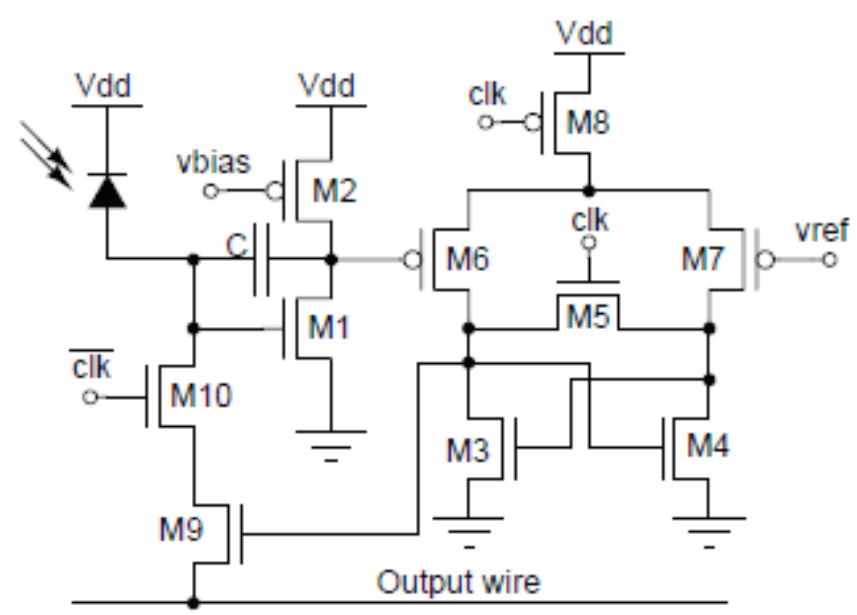

Fig. (36). Schematic diagram of each pixel.

The circuit consists in three main sections: the integrator, composed by $\mathrm{M}_{1}, \mathrm{M}_{2}$ and $\mathrm{C}$, the comparator, composed by transistors $\mathrm{M}_{3}$ to $\mathrm{M}_{8}$ and the 1 bit digital to analog converter, composed by $\mathrm{M}_{9}$ and $\mathrm{M}_{10}$.

\subsubsection{Integrator}

The integrator is based on a common-source amplifier, where the capacitor establishes a negative feedback, forming a Miller integrator, whose capacitance multiplication is given by:

$$
C_{M}=C\left(1-A_{v}\right) \text {, }
$$

where $A_{v}$ is the gain of the common-source amplifier. With this technique, good performances are reached with capacitors as small as $100 \mathrm{fF}$.

Fig. (37) shows the output voltage of the integrator for photodiode currents of $10 \mathrm{nA}$ and $100 \mathrm{nA}$. The linearity of these curves results in coefficients of Pearson -0.9999979 and -0.999999929 respectively, that is, very close to 1 . Notices that the integrator does not need to be linear for the sigma-delta converter work correctly. However, with a linear integrator, the signal to noise ratio is increased $[84,85]$.

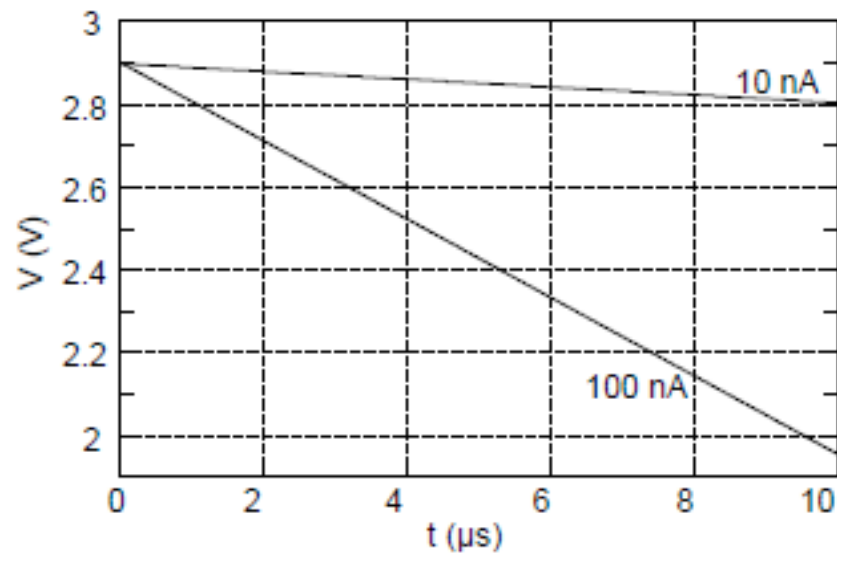

Fig. (37). Output voltage the integrator for input currents of $10 \mathrm{nA}$ and $100 \mathrm{nA}$.

The bias voltage of $\mathrm{M}_{2}\left(V_{\text {bias }}\right)$ is supplied by current mirror configuration, placed outside of the matrix and common to all pixels.

\subsubsection{Comparator}

In the comparator circuit, $\mathrm{M}_{6}$ and $\mathrm{M}_{7}$ form a differential pair that amplifies the difference between the output voltage of the integrator and $V_{\text {ref. }}$. The difference is stored in the pair $\mathrm{M}_{3}$ and $\mathrm{M}_{4}$, which forms a latch, in the negative transitions of clock. The state of this latch is kept constant while $\mathrm{M}_{5}$ is not conducting, that is, while the clock is at the low level. When the clock changes to the high level, $\mathrm{M}_{8}$ disconnects the circuit, reducing the power consumption.

The performance of the comparator is shown in Fig. (38), for a $V_{\text {ref }}$ of $1.5 \mathrm{~V}$ and a random input voltage, $v_{i n}$. In this figure it can be observed that to each negative transition of clock, the output voltage of the comparator goes to the high logical level if $v_{i n}<V_{\text {ref }}$ and goes to the low logical level if $v_{i n}>V_{\text {ref. }}$. In this figure, the clock signal has a period of $1 \mu \mathrm{s}$ and a duty-cycle of $99 \%$, originating a duty-cycle of $1 \%$ in the output signal.

In the circuit of Fig. (36) it is convenient to use a clock signal with a very high duty-cycle, for two basic reasons: firstly, during the comparison (clock at the low level), the input signal must be approximately constant. Secondly, this procedure allows the sharing of the output wire, as it will be seen in the next paragraphs. 


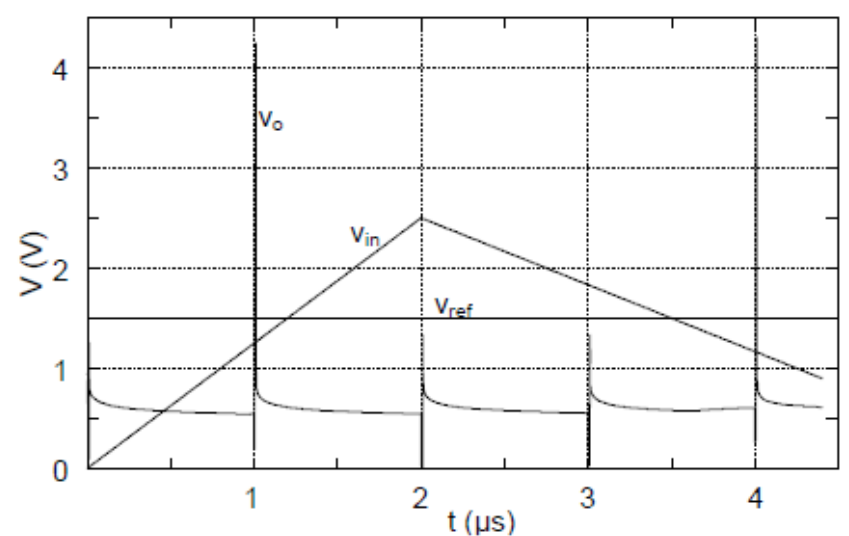

Fig. (38). Output waveform of the comparator, for a random input voltage, $v_{i n}$ and $V_{\text {ref }}=1.5 \mathrm{~V}$. The clock signal has a period of $1 \mu \mathrm{s}$ and a duty-cycle of $99 \%$.

\subsubsection{Bit Digital to Analog Converter}

The 1 bit digital to analog converter is composed by $\mathrm{M}_{9}$ and $\mathrm{M}_{10}$, which perform a logical AND operation between the output signal of the comparator and the complementary of the clock signal. Therefore, when clock is at the low level (the complementary is high) and the output signal of the comparator is high, the capacitor $\mathrm{C}$ is discharged and a current pulse is injected in the output wire.

The output signal of each sigma-delta converter appears under the form of a current, obtained from the discharge of the capacitor. This output signal is only activated at the low level of clock, being in a high impedance state in the remaining time. This means that with a clock with high dutycycle, the possible number of pixels in each line can be bigger, being enough that each column receives its clock pulse shifted in time. Additionally, with this technique it is possible to share the output wire by all pixels of the same line. For example, a clock signal with duty-cycle of $99.9 \%$, allows a matrix with 1000 columns to share the same output wire of each line, since each column is active during $0.1 \%$ of the clock period.

Fig. (39) shows the waveform the output signal of the comparator, for input currents of 67nA Fig. (39a) and 130nA Fig. (39b).

\section{CURRENT \& FUTURE DEVELOPMENTS}

The main goal of this review was to present the main issues related to the design and fabrication of X-ray detectors based in scintillators. Mats et al. described the present invention relates to a device with high competence and high spatial resolution for detection of individual x-rays [86]. The first step necessary to detect the X-rays is that its energy must be absorbed by some material, that is, it is necessary that some interaction between X-rays and matter occurs. After the X-ray energy being absorbed, there are some processes of converting it into electric signals, namely based in photoconductive materials, in scintillators or in thermal processes. From that processes, the detectors based on scintillators, have the best characteristics in terms of performance. The scintillators, when absorbing X-rays, emit

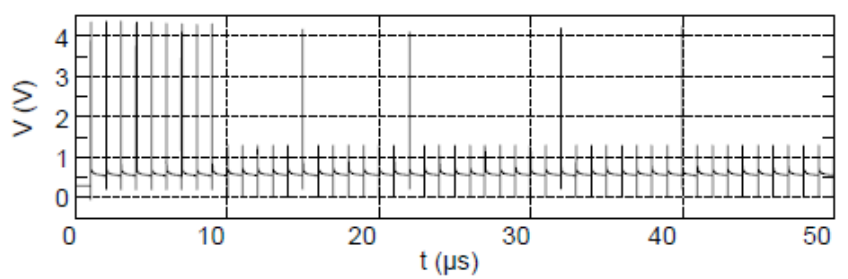

(a)

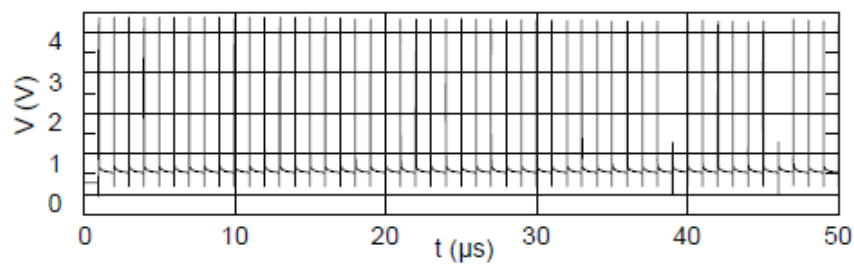

(b)

Fig. (39). Output waveforms of the comparator for input currents of (a) $67 \mathrm{nA}$; (b) $130 \mathrm{nA}$.

visible light, which in turn, must reach the photodetectors so that it is converted into electric signals. So, it is necessary an interface between both, which ideally does not introduce losses. The visible light is absorbed by the photodetectors and converted into electric currents. The electric currents are finally converted into digital signals by the interface electronics, which in this case, the first-order sigma-delta converters associated with DPS architectures seems to be a very promising solution.

\section{CONFLICT OF INTEREST}

The authors declare no conflict of interest.

\section{ACKNOWLEDGEMENTS}

The authors thank the Portuguese FCT for financial support. JGR thanks the FCT for the SFRH/BSAB/1014/ 2010 grant.

\section{REFERENCES}

[1] The Columbia electronic encyclopedia. Columbia University Press, USA 2000

[2] Mei, E.,Norey, C.,Masino, J. A system and method for simultaneously obtaining a plurality of images in an imaging system. WO2010077205 (2010).

[3] Selman J. The fundamentals of X-rays and radium physics. Charles C Thomas Publisher, Illinois USA, $8^{\text {th }}$ ed. 1994.

[4] Inazuru, T. X-ray tube and X-ray source. US7773726 (2010).

[5] Grupen C. Physics of particle detection. arXiv:physics/9906063v1 [physics.ins-det], 1999.

[6] Marmier P, Sheldon E. Physics of nuclei and particles. 1 Academic Press, New York 1969.

[7] Knoll G. F. Radiation Detection and Measurement. Wiley, New york, USA, $3^{\text {rd }}$ ed. 2000.

[8] Grupen C. Particle Detectors. Cambridge University Press, 1996.

[9] Rossi B. High Energy Particles. Prentice-Hall, 1952.

[10] Faw RE, Shultis JK. Radiological assessment sources and exposures. PRT Prentice-Hall, Inc., 1993.

[11] Hayward E. Photonuclear reactions. NBS Monograph 118, U.S. National Bureau of Standards, 1970. 
[12] Fuller EG, Hayward E. Photonuclear Reactions. Dowden, Hutchinson and Ross, Stroudsburg Pennsylvania, 1976.

[13] Dietrich SS, Berman BL. Atlas of photoneutron cross sections obtained with monoenergetic photons. In At Data Nucl Data Tables 1988; 38: 199-338.

[14] Hubbell JH. Photon cross sections, attenuation coefficients, and energy absorption coefficients from $10 \mathrm{keV}$ to $100 \mathrm{GeV}$. NSRDSNBS 29, U.S. National Bureau of Standards, 1969.

[15] Hubbell JH. Photon mass attenuation and energy-absorption coefficients from $1 \mathrm{keV}$ to $20 \mathrm{MeV}$. Int J Appl Radiat Isot 1982; 33:1269-90.

[16] Gimm HA, Hubbell JH. Total photon absorption cross section measurements, theoretical analysis and evaluations for energies above $10 \mathrm{MeV}$. NBS Techn Note 968, U.S. National Bureau of Standards, 1978.

[17] Schumacher M. Delbrueck scattering. Radiat Phys Chem 1999; 56: $1-111$.

[18] Lee RN, Milstein AI, Strakhovenko VM. Simple analytical representation for the high-energy delbrück scattering amplitudes. Budker inp 99-6, Siberian Branch of Russian Academy of Science Budker Institute of Nuclear Physics, Novosibirsk, 1999.

[19] Sakellaris T, Spyrou G, Tzanakos G, Panayiotakis G. Monte Carlo simulation of primary electron production inside an a-selenium detector for X-ray mammography: physics. Phys Med Biol 2005; 50: 3717-38.

[20] Zentaia G, Schieberb M, Partain L, Pavlyuchkova R, Proano C. Large area mercuric iodide and lead iodide X-ray detectors for medical and non-destructive industrial imaging. J Crystal Growth 2005; 275: e1327 - e31.

[21] Eisen Y, Shor AI. CdTe and CdZnTe X-ray and gamma-ray detectors for imaging systems. IEEE Trans Nucl Sci 2004; 51: $1191-8$.

[22] Sun GC, Samic H, Bourgoin JC, Chambellan D, Gal O, Pillot P. A comparison between $\mathrm{GaAs}$ and $\mathrm{CdTe}$ for $\mathrm{X}$-ray imaging. IEEE Trans Nucl Sci 2004; 51: 2400 - 4.

[23] Rocha JG, Goncalves LM, Lanceros-Mendez S. Flexible X-ray detector based on the seebeck effect. Proc Transducers 2009, Denver, Colorado, USA, 21-25 Junho 2009.

[24] Rocha, J.G., Lanceros-Mendez, S. X-ray imaging matrix with light guides and intelligent pixel sensors, radiation or high energy particle detector devices that contain it, its fabrication process and its use. WO2007046010 (2007).

[25] Dubaric E, Fröjdh C, Nilsson HE, Petersson CS. Resolution noise and properties of scintillator coated X-ray detectors. Nucl Instr Meth Phys Res 2001; 466: 178-82.

[26] Irsigler R, Andersson J, Alverbro J, Borglind J, Fröjdh C, Helander P, Manolopoulus S, Martijn H, O’Shea V, Smith K. X-ray imaging using a $320 \times 240$ hybrid GaAs pixel detector. IEEE Trans Nucl Sci 1999; 46: 507-12.

[27] Workman A, Brettle DS. Physical performance measures of radiographic imaging systems. Dentomaxillofacial Radiol 1997; 26: 139-46.

[28] Tate MW, Eikenberry EF, Barna SL, Wall ME, Lowrance JL, Gruner SM. A large-format high-resolution area X-ray detector based on a fiber-optically bonded charge-coupled device (CCD). J Appl Crystallogr 1995; 28: 196-205.

[29] Boone JM, Fewell TR, Jennings RJ. Molybdenum rhodium and tungsten anode spectral models using interpolating polynomials with application to mammography. Med Phys 1997; 24: 1863 874 .

[30] Fujieda I, Cho G, Drewery J, Gee T, Jing T, Kaplan SN, PerezMendez V, Wildermuth D, Street RA. X-ray and charged particle detection with $\mathrm{CsI}(\mathrm{Tl})$ layer coupled to a $\mathrm{Si}: \mathrm{H}$ photodiode layers. IEEE Trans Nucl Sci 1991; 38: 255 - 62.

[31] Valentine J, Wehe D, Knoll G, Moss C. Temperature dependence of absolute CsI(Tl) scintillation yield. Proc IEEE Nucl Sci Symp Conf Rec 1991; 176-82.

[32] Gruner SM, Tate MW, Eikenberry EF. Charge-coupled device area X-ray detectors. Rev Sci Instrum 2002; 73: 2815-42.

[33] Swank RK. Absorption and noise in X-ray phosphors. J Appl Phys 1973; 44(9): 4199-203.

[34] Ruetten, W., Kiewitt, R., Wischhusen, O. X-ray detector. WO2010058369 (2010)

[35] Allier CP, Hollander RW, van Eijk CWE, Sarro PM, de Boer M, Czirr JB, Chaminade JP, Fouassier C. Thin photodiodes for a neutron scintillator siliconwell detector. IEEE Tran Nucl Sci 2001; 48:1154-7.

[36] Kleimann P, Linnros J, Frojdh C, Petersson CS. An X-ray imaging pixel detector based on a scintillating guides screen. IEEE Tran Nucl Sci 2000; 47: 1483-6.

[37] Badel X, Linnros J, Kleimann P, Norlin B, Koskiahde E, Valpas K, Nenonen S, Petersson CS, Fröjdh C. Metallized and oxidized silicon macropore arrays filled with a scintillator for CCD-based Xray imaging detectors. IEEE Trans Nucl Sci 2004; 51: 1001-5.

[38] Badel X, Galeckas A, Linnros J., Kleimann P., Fröjdh C., Petersson CS. Improvement of an X-ray imaging detector based on a scintillating guides screen. Nucl Instrum Methods Phys Res A 2002; 487: 129-35.

[39] Pedrotti FL, Pedrotti LS. Introduction to optics, 2nd ed. Englewood Cliffs, NJ: Prentice-Hall, 1996.

[40] Gordon SP, Gordon FS. Contemporary Statistics-A Computer Approach. Singapore: McGraw-Hill, 1994.

[41] Hjelm M, Norlin B, Nilsson HE, Fröjdh C, Badel X. Monte Carlo simulation of the imaging properties of scintillator-coated X-ray pixel detectors. Nucl Instrum Methods Phys Res A 2003; 509: 7685 .

[42] Jansen H, de Boer M, Elwenspoek M. Black silicon method VI: High aspect ratio trench etching for MEMS applications. Proc IEEE Micro Electro Mechanical Syst (MEMS), 1996; 250-7.

[43] Microchem. (s.d.). Processing Guidelines for: SU-8 2100 \& SU-8 2150. Obtained in 10 July 2009, from SU-8 2000 Permanent Epoxy Negative Photoresist: www.microchem.com.

[44] Frazier AB, Allen MG. Uses of Electroplated Aluminum for the Development of Microstructures and Micromachining Processes. J Microelectromechanical Syst. 1997; 6: 91-8.

[45] Fromberg W, Donaldson FA. Electroplating with aluminum. Adv Mater Process 1996; 149: 33-5.

[46] Gileadi E, Capuano G. High purity aluminum electroplating. Adv Mater Process. 1989; 135: 14-8.

[47] Van de Berg JF, Van Dijk GA, Van de Leest RE. Room temperature electroplating of aluminum. Metal Finishing 1985; 83: $15-8$.

[48] Chakraborty, S. X-ray detector. US7756250 (2010).

[49] Rocha JG, Ramos NF, Lanceros-Mendez S, Wolffenbuttel RFJ, Correia H. CMOS X-rays detector array based on scintillating light guides. Sensors Actuators A 2004; 110: 119-23.

[50] Badel X, Galeckas A, Linnros J, Kleimann P, Frojdh C, Petersson CS. Improvement of an X-ray imaging detector based on a scintillating guides screen. Nuclear Instruments Methods A 2002; 487: 129-35.

[51] Ananenko A, Fedorov A, Mateychenko P, Tarasov V, Vidaj Y. Structure and scintillation properties of $\mathrm{CsI}(\mathrm{Tl})$ epitaxial layers. Appl Surface Sci 2004; 236: 186-91.

[52] Jing T, Goodman CA, Drewery J, Cho G, Hong WS, Lee H, Kaplan SN, Mireshghi A, Perez-Mendez V, Wildermuth D. Amorphos silicon pixel layer with caesium iodide converters for medical radiography. IEEE Trans Nucl Sci. 1994; 41: 903-9.

[53] Lorenz RA, Osborne MF, Collins JL, Manning SR, A. Malinauskas P. Behavior of Iodine, Methyl Iodide, Cesium Oxide, and Cesium Iodide in Steam and Argon. technical report, Oak Ridge National Laboratory, 1976.

[54] Cha BK, Shin JH, Bae JH, Lee C, Chang S, Kim HK, Kim CK, Cho G. Scintillation characteristics and imaging performance of CsI: Tl thin films for X-ray imaging applications. Nucl Instr Meth Phys Res A 2009; 604: 224-8.

[55] Ananenko A., Fedorov A, Lebedinsky A, Mateychenko P, Tarasov V, Vidaj Y. Structural dependence of CsI(Tl) film scintillation properties. Semiconductor Phys, Quantum Electron Optoelectron 2004; 7: 297-300.

[56] Vydai YT, Tsirlin YA, Chaikovskii EF. Dependece of the scintillation characteristics of thin film $\mathrm{CsI}(\mathrm{Tl})$ detectors upon the thallium. J Applied Spectroscopy 1975; 22: 344-6.

[57] Fujieda I, Cho G, Drewery J, Gee T, Jing T, Kaplane SN, PerezMendez V, Wildermuth D, Street RA. X-ray and Charged Particle Detection with CsI(T1) Layer Coupled to a-Si:H Photodiode Layers. IEEE Trans Nucl Sci 1991; 38: 255-62.

[58] Nagarkar VV, Gupta TK, Miller S, Klugerman Y, Squillante MR. Structured $\mathrm{CsI}(\mathrm{Tl})$ scintillators for X-ray imaging applications. IEEE Trans Nucl Sci 1998; 44: 492-6. 
[59] Schotanus P, Kamermans R, Dorenbos P. Scintillation characteristics of pure and T1-doped CsI crystals. IEEE Trans Nucl Sci 1990; 37: 177-82.

[60] Moini A. Vision Chips. Kluwer Academic Publishers, 2000.

[61] de Lima Monteiro DW. CMOS-based integrated wavefront sensor. $\mathrm{PhD}$ thesis, Delft University of Technol 2002.

[62] Wehr RM, Richards JA, Adair TW. Physics of the atom. Addison Wesley, 4th ed, 1984

[63] Delbruck T. Investigation of analog VLSI visual transduction and motion processing. $\mathrm{PhD}$ thesis, California Institute of Technology, 1993.

[64] Wong H. Technology and device scaling considerations for CMOS imagers. IEEE Trans Electr Dev 1996; 43: 2131-42.

[65] Wong HP, Chang RT, Crabbe E, Agnello PD. CMOS active pixel image sensor fabricated using a $1.8-\mathrm{V}, 0.25 \mu \mathrm{m}$ CMOS technology. IEEE Trans Electron Devices 1998; 45: 889-94.

[66] Spahn, M. CMOS flat X-ray detector. US7622719 (2009).

[67] Koike N, Takemoto I, Satoh K, Hanamura S, Nagahara S, Kubo M. MOS Area sensor: Part I - Design Consideration and Performance of an n-p-n structure 484 x 384 Element Color MOS Imager. IEEE Trans Electron Devices 1980; 27: 1682- 7.

[68] Ohaba S, Nakai M, Ando H, Hanamura S, Shimada S, Satoh K, Takahashi K, Kubo M, Fujita T. MOS Area Sensor: Part II- LowNoise MOS Area Sensor with Antiblooming Photodiodes. IEEE J Solid-State Circuits 1980; 15: 747-52.

[69] Noda M, Imaide T, Kinugasa T, Nishimura R. A solid state color video camera with a horizontal readout MOS imager. IEEE Trans Consumer Electron 1986; 32: 329-36.

[70] Kawahito S, Yoshida M, Sasaki M, Umehara K, Miyazaki D, Tadokoro Y, Murata K, Doushou S, Matsuzawa A. A CMOS image sensor with analog two-dimensional DCT-based compression circuits for one-chip cameras. IEEE J Solid-State Circuits 1997; 32: 2030-41.

[71] Miyazawa T, Nishizawa S, Uehara M, Nakano Y, Nakamura S, Tanaka K, Horiuchi T, Takemoto K. TSL solid-state imager. Proc TV Eng Soc Meeting Japan, 1986; 59-60.

[72] Miyatake S, Miyamoto M, Ishida K, Miramoto T, Masaki Y, Tanabe H. Transversal-readout architecture for CMOS active pixel image sensors. IEEE Trans Electron Devices 2003; 50: 121-9.
[73] Kleinfelder S, Lim S, Liu X, El Gamal A. A 10000 Frames/s CMOS Digital Pixel Sensor. IEEE J Solid-State Circuits 2001; 36: 2049-59.

[74] Sawan M, Trepanier A, Trepanier JL, Audet Y, Ghannoum R. A new CMOS multimode digital pixel sensor dedicated to an implantable visual cortical stimulator. Analog. Integr Circ Sig Process 49: 2006; 187-97.

[75] Rocha JG, Dias RA, Goncalves L, Minas G, Ferreira A, Costa CM, Lanceros-Mendez S. X-ray image detector based on light guides and scintillators. IEEE Sensors J 2009; 9: 1154-9.

[76] Ohta J. Smart CMOS image sensors and applications. CRC Press, Taylor \& Francis Group, LLC, USA, 2008.

[77] Yang W. A wide-dynamic-range, low power photosensor array. Proc IEEE Solid-State Circ Conf, San Francisco, CA, USA, 1994; 230-1.

[78] Battaglia M, Bisello D, Contarato D, Denes P, Giubilato P, Glesener L, Mattiazzo S, Vu C. Monolithic pixel sensors in deepsubmicron SOI technology with analog and digital pixels. Nucl Instr Meth Phys Res A, 2009; 604: 380-4

[79] Noble P. Self-scanned silicon image detector arrays. IEEE Trans Electron Devices 15: 1968; 202-9.

[80] Mandl W, Rutschow C. All digital monolithic scanning readout based on sigma-delta analog to digital conversion. Proc SPIE 1992; 1684: 239-46.

[81] Fowler B, Gamal A, Yang DXD. A CMOS area image sensor with pixel-level A/D conversion. Proc Solid-State Circ Conf, San Francisco, CA, USA, 1994; 226-7.

[82] Fowler, B., Gamal, A. CMOS image sensor with pixel level A/D conversion. US5461425 (1995).

[83] Mandl WJ, Kennedy JJ, Chu M. MOSAD IR focal plane array development. Proc SPIE, 2745: 1996; 90-8.

[84] Candy JC, Temes GC, editors, "Oversampled delta-sigma data converters. IEEE Press, New York, 1992.

[85] Fernandes A, Cardoso VVF, Rocha JG, Cabral J, Minas G. Smartoptical detector CMOS array for biochemical parameters analysis in physiological fluids. IEEE Trans Industrial Electron 2008; 55: 3192-200.

[86] Danielsson, M., Tibbelin, S. X-ray detector, a corresponding X-ray imaging device and a method for improving the resolution of a scintillator-based X-ray detector. US20100096558 (2010). 